\title{
Baroclinic Waves with Parameterized Effects of Moisture Interpreted Using Rossby Wave Components
}

\author{
Hylke de Vries,* John Methren, and Thomas H. A. Frame \\ Department of Meteorology, University of Reading, Reading, United Kingdom \\ BRIAN J. HOSKINS \\ Department of Meteorology, University of Reading, Reading, and Grantham Institute for Climate Change, \\ Imperial College, London, United Kingdom
}

(Manuscript received 11 December 2009, in final form 26 February 2010)

\begin{abstract}
A theoretical framework is developed for the evolution of baroclinic waves with latent heat release parameterized in terms of vertical velocity. Both wave-conditional instability of the second kind (CISK) and large-scale rain approaches are included. The new quasigeostrophic framework covers evolution from general initial conditions on zonal flows with vertical shear, planetary vorticity gradient, a lower boundary, and a tropopause. The formulation is given completely in terms of potential vorticity, enabling the partition of perturbations into Rossby wave components, just as for the dry problem. Both modal and nonmodal development can be understood to a good approximation in terms of propagation and interaction between these components alone. The key change with moisture is that growing normal modes are described in terms of four counterpropagating Rossby wave (CRW) components rather than two. Moist CRWs exist above and below the maximum in latent heating, in addition to the upper- and lower-level CRWs of dry theory. Four classifications of baroclinic development are defined by quantifying the strength of interaction between the four components and identifying the dominant pairs, which range from essentially dry instability to instability in the limit of strong heating far from boundaries, with type-C cyclogenesis and diabatic Rossby waves being intermediate types. General initial conditions must also include passively advected residual PV, as in the dry problem.
\end{abstract}

\section{Introduction}

Case studies indicate that condensation of water vapor often plays an important role in baroclinic life cycles (e.g., Gyakum 1983; Wernli et al. 2002). Structural changes, a more rapid intensification and propagation, and a reduction in scale of the ascent region are well-documented differences relative to cyclone development in the absence of moisture (e.g., Emanuel et al. 1987; Kuo et al. 1991; Moore and Montgomery 2004). Despite this evidence, it is still a challenge to satisfactorily underpin these differences with theory. One of the difficulties is the parameterization of the link between latent heat release and

\footnotetext{
* Additional affiliation: Royal Netherlands Meteorological Institute, De Bilt, Netherlands.

Corresponding author address: Dr. Hylke de Vries, P.O. Box 201, KNMI, De Bilt 3730 AE, Netherlands.

E-mail: hylke.de.vries@knmi.nl
}

dynamical variables. The two main approaches for baroclinic waves to date both link latent heat release to saturation in ascending air masses, either based on the large-scale ascent rate at every point (Emanuel et al. 1987; Montgomery and Farrell 1991; Whitaker and Davis 1994, hereafter WD94) or associated with ascent rate at a specified cloud base with an assumed heating profile representing deep convection (Mak 1982; Snyder and Lindzen 1991, hereafter SL91; Parker and Thorpe 1995).

Since diabatic cooling in the unsaturated descending air (associated with radiative transfer or evaporation of precipitation) typically does not occur as rapidly, the feedback between condensational heating and the baroclinic wave dynamics is intrinsically asymmetrical and nonlinear. In contrast, much of the theory on "dry" baroclinic instability is firmly rooted in linear wave theory and concepts such as propagating Rossby waves (RWs) and their interactions in geophysical shear flows. There are two well-known necessary conditions that determine whether a given zonal flow could support exponentially 
amplifying disturbances, known as growing normal modes (gNMs). The first states that the meridional potential vorticity (PV) gradient $\bar{q}_{y}$ changes sign at least once within the domain (Charney and Stern 1962). The second condition states that $\bar{q}_{y}$ should be positively correlated with the zonal flow (Fjørtoft 1950). Both criteria are derived for dry adiabatic dynamics. It is an open question what are the necessary and sufficient conditions for "moist" baroclinic instability. Partly, this question is still unanswered because the realism of linear moisture parameterizations is debated. Nevertheless, in this paper the moisture parameterizations are taken mostly in their linearized forms. This linearization can be justified in the weak-heating limit when large-scale adiabatic motion dominates. For higher diabatic heating rates the wave structure becomes distorted and is no longer purely sinusoidal in the zonal direction (e.g., Emanuel et al. 1987; Parker and Thorpe 1995). From the perspective of Fourier decomposition, the linearized theory accounts for the dominant effect of diabatic heating occurring at wavenumber $k$ on the dynamics of wave- $k$. Interactions between waves of differing wavenumber are ignored at leading order.

The principal objective of this paper is to construct a framework for the analysis of linear quasigeostrophic (QG) baroclinic wave development from general initial conditions in the presence of simple moisture parameterizations. Two parameterizations are considered; both relate diabatic heating to vertical velocity and are referred to as large-scale rain (LSR) (Emanuel et al. 1987) and wave-conditional instability of the second kind (wave-CISK ${ }^{1}$ ) or cumulus convection (CCV) (Mak 1982). The framework uses Rossby wave components as its basis. RW components are defined as untilted waves in PV. PV is used because of its well-known Lagrangian conservation property as well as its invertibility (Hoskins et al. 1985). Moreover, in absence of moisture, there exists a framework for interpreting modal baroclinic instability on zonal jets in terms of two mutually reinforcing, phase-locking counterpropagating Rossby waves (CRWs) (Heifetz et al. 2004a, hereafter H04). CRWs have a particular untilted PV structure that can be obtained by linear superposition of a gNM and its complex conjugate (H04). They are direct generalizations of the well-known boundary edge waves of the Eady (1949) model (Davies and Bishop 1994) allowing for nonzero interior PV gradient.

CRW theory can explain the growth from infinitesimal perturbations that have been created by meridional displacements of PV contours from a zonally symmetric

\footnotetext{
${ }^{1}$ Wave-CISK was introduced in Lindzen (1974).
}

basic state. However, PV anomalies can have a different origin and could, for instance, be remnants of earlier diabatic processes. Recently, De Vries et al. (2009, hereafter V09) have shown that the transient linear development from general initial conditions can also be compactly described by considering the evolution of these two CRWs plus a passively advected remainder. One of the reasons why this so-called passively advected residual PV (PARPV) approximation works, is that it captures three essential growth mechanisms available to the dry dynamics (De Vries and Opsteegh 2007; V09): (i) shear instability, the interactions between CRWs; (ii) resonance, the excitation of CRWs by the passive remainder PV; and (iii) the Orr mechanism, the transient growth arising from the differential advection of interior PV structures by the shear flow, first described in Orr (1907).

Realistically, however, diabatic heating occurs while the wave develops. Thus, the instantaneous PV disturbance field in a moist atmosphere has three different origins:

1) Displacement, $\mathrm{PV} q^{(d)}$, which is obtained through advection of the basic-state PV contours by the perturbation meridional wind;

2) Diabatic, $\mathrm{PV} q^{(m)}$, resulting from the "active" diabatic processes; and

3) Passive, $\mathrm{PV} q^{(p)}$, which describes "remnant" $\mathrm{PV}$ from past diabatic processes. Passive PV is simply passively advected with the zonal flow.

The above partitioning of the PV disturbance field is attractive because of its conceptual simplicity and similar approaches have been used in diagnosis of numerical weather prediction models (e.g., Gray 2006). Many authors discuss the PV dynamics of moist baroclinic waves as a qualitative tool to understand better the unstable wave's vertical structure (e.g., Montgomery and Farrell 1991; SL91; Raymond and Jiang 1990; WD94; Parker and Thorpe 1995; Moore and Montgomery 2004). To the authors' knowledge, however, no attempt has yet been made to formulate a general framework for the PV analysis of moist baroclinic initial value problems that is applicable to a wide range of quasigeostrophic models, with or without interior PV gradients. Such a framework is presented in this paper, extending V09 by including diabatic heating and the generation of "diabatic PV anomalies" $q^{(m)}$. RW components will be defined based on the three different types of PV anomalies described above, and their interactions will be analyzed.

Section 2 details key aspects of the model dynamics and the moisture parameterizations. Two models in which the RW components assume a particularly simple form are discussed in section 3 . In section 4 a tropopause is added 
in the form of a rigid lid (Mak 1994). Four classifications of baroclinic development are defined by quantifying the strength of interaction between the four components and identifying the dominant pairs, ranging from essentially dry instability to instability in the limit of strong heating far from boundaries, with type-C cyclogenesis and diabatic Rossby waves being intermediate types. The generalization of the theory to include a planetary vorticity gradient, large-scale rain parameterization, and more general initial conditions are given in sections 5-7. Conclusions are presented in section 8 .

\section{Model description}

\section{a. Quasigeostrophic potential vorticity dynamics}

The QG evolution of perturbations to a zonal flow in the presence of a diabatic heating $\dot{\Theta}$ can be described by the QG PV equation

$$
\frac{\partial q}{\partial t}+\bar{u} \frac{\partial q}{\partial x}+v \frac{\partial \bar{q}}{\partial y}=S, \quad S=\frac{1}{\bar{\rho}} \frac{\partial}{\partial z}\left(\frac{\bar{\rho} f_{0}^{2} \dot{\Theta}}{N^{2}}\right),
$$

where $q=\mathcal{L}_{\psi}[\psi]=\nabla_{h}^{2} \psi+\bar{\rho}^{-1} \partial_{z}\left(\bar{\rho} f_{0}^{2} N^{-2} \partial_{z} \psi\right)$ is the perturbation PV, $\nabla_{h}^{2}=\partial_{x}^{2}+\partial_{y}^{2}, v=\partial \psi / \partial x$ is the geostrophic meridional wind, $\psi$ is the perturbation streamfunction, and $\bar{\rho}(z)$ is a reference density profile. Basic-state quantities carry a bar. The interior mean PV gradient $\bar{q}_{y}$ is given by

$$
\frac{\partial \bar{q}}{\partial y}=\beta-\frac{\partial^{2} \bar{u}}{\partial y^{2}}-\frac{1}{\bar{\rho}} \frac{\partial}{\partial z}\left(\frac{\bar{\rho} f_{0}^{2}}{N^{2}} \frac{\partial \bar{u}}{\partial z}\right),
$$

where $\beta$ is the planetary vorticity gradient and $N^{2}$ is the buoyancy frequency. We restrict to two-dimensional perturbations although meridional wavenumbers are easily included. The equations are made nondimensional using a height scale $H=\left(f_{0} / N\right)^{2} \Lambda / \beta \sim 15 \mathrm{~km}$, where $f_{0}$ and $\beta$ values are set to those for the earth at $45^{\circ} \mathrm{N}$ and $\Lambda$ is the shear. The horizontal scale $L=\left(N / f_{0}\right) H$, time scale $N /\left(f_{0} \Lambda\right)$, and other scalings are as in Heifetz et al. (2004b). Rigid lids may be prescribed at $z_{b}$ and $z_{t}$ and are taken into account by setting the vertical velocity $w$ to zero in the thermodynamic equation

$$
\frac{\partial \theta}{\partial t}+\bar{u} \frac{\partial \theta}{\partial x}+v \frac{\partial \bar{\theta}}{\partial y}=0, \quad z=\left(z_{b}, z_{t}\right)
$$

where $\theta=\partial \psi / \partial z$ is the potential temperature rescaled by the constant $g /\left(f_{0} \theta_{0}\right)$ such that thermal wind balance reduces to $\bar{\theta}_{y}=-\partial \bar{u} / \partial z$. Heating at lids as in Mak and Bannon (1984) is not considered.

\section{b. Moisture parameterization schemes}

The simplest parameterization relates heating to vertical velocity on the premise that ascending air saturates rapidly and experiences latent heat release. Two types of parameterization have been investigated: wave-CISK (Mak 1982; Craig and Cho 1988; SL91; Parker and Thorpe 1995), which is referred to as CCV, and LSR, as discussed for instance by Emanuel et al. (1987) and WD94.

The wave-CISK (CCV) parameterization is based on the assumption that ascending air saturates and precipitates above level $z_{*}$ (usually taken to be near $900 \mathrm{hPa}$ ), producing diabatic heating $\dot{\Theta}_{\mathrm{CCV}}$ in a column vertically above $z_{*}$ with a particular heating profile $h(z)$, normalized such that $\int h(z) N^{2} d z=1$. If we assume unsaturated adiabatic descent, the diabatic heating takes the following conditional form:

$\dot{\Theta}_{\mathrm{CCV}}(x, z, t)=\epsilon_{\mathrm{CCV}} h(z)\left[w\left(x, z_{*}, t\right)+\left|w\left(x, z_{*}, t\right)\right|\right]$,

where $w\left(z_{*}, t\right)$ is the vertical velocity at level $z_{*}$, and $\epsilon_{\mathrm{CCV}}$ is the heating intensity parameter. SL91 have argued that $\epsilon_{\mathrm{CCV}}$ is likely to be proportional to the depth of the heating layer, $\Delta z=z_{2}-z_{1}$ (a deeper cloud requires more intense diabatic heating), and that $\epsilon_{\mathrm{CCV}} / \Delta z=O(1)$ is consistent with (idealized) estimates of the large-scale budgets of heat and moisture. However, there is no implied form for the structure of $h(z)$. Mathematically simplest is to make $h(z)$ a top-hat; that is, $N^{2} h(z)=$ $\left[H\left(z-z_{1}\right)-H\left(z_{2}-z\right)\right] / \Delta z$, where $H(z)$ is the Heaviside step function (Mak 1982; SL91). Another option is a triangularly shaped heating profile (Craig and Cho 1988; Parker and Thorpe 1995).

The LSR parameterization assumes that the diabatic heating rate is proportional to the local vertical velocity $w(z)$ [rather than to $w\left(z_{*}\right)$ as in wave-CISK]:

$$
\dot{\Theta}_{\mathrm{LSR}}(x, z, t)=\epsilon_{\mathrm{LSR}} r(z)[w(x, z, t)+|w(x, z, t)|],
$$

where the function $r(z)$ is a moisture profile. Emanuel et al. (1987) used constant $r(z)$ for reasons of simplicity. WD94 argued that a thermodynamically more consistent profile of $r(z)$ will decay almost linearly with height in the lower troposphere and more rapidly aloft, such that $r(z)$ is zero at the tropopause. Moore and Montgomery (2004) assumed a linear decrease of $r(z)$ with height [the notation $r(z)$ differs from that used in the papers cited above].

As described in the introduction, the conditionality on the sign of $w$ renders the parameterizations intrinsically nonlinear. This paper describes the simpler problem where symmetry between ascent and descent is enforced such that diabatic cooling occurs in descending air [i.e., dropping the $|w|$ term from (5)]. The linearization 
guarantees that a perturbation with a single wavenumber $k$ does not interact with others. The more complex problem of conditional diabatic heating is left to a future paper (Frame et al. 2010, unpublished manuscript).

\section{c. Quasigeostrophic omega equation}

The evolution of perturbations in balanced adiabatic flows can be described fully by the PV advection equation (1) together with the PV inversion equation, $q=$ $\mathcal{L}_{\psi}[\psi]$, and the thermodynamic equation on the boundaries (3). However, in moist problems when latent heating depends on vertical velocity, it is necessary also to consider the omega equation. In the presence of diabatic heating, the QG omega equation can be written as

$$
\mathcal{L}_{w}[w]=F^{(d)}+F^{(h)},
$$

where $\mathcal{L}_{w}[w]=N^{2} \nabla_{h}^{2} w+\partial_{z}\left[\bar{\rho}^{-1} \partial_{z}(\bar{\rho} w)\right]$. The two terms on the right-hand side of (6) can be thought of as "forcings" driving the vertical circulation (Hoskins et al. 2003). The first term is

$$
\begin{aligned}
F^{(d)}= & \frac{\partial}{\partial z}\left[(\overline{\mathbf{u}}+\mathbf{u}) \cdot \nabla_{h}(\bar{\xi}+\xi)+\beta v\right] \\
& -\nabla_{h}^{2}\left[(\overline{\mathbf{u}}+\mathbf{u}) \cdot \nabla_{h}(\bar{\theta}+\theta)\right],
\end{aligned}
$$

where $\mathbf{u}=(u, v)$ and $\xi=\nabla_{h}^{2} \psi$ is the relative vorticity. After linearization about a zonal basic state, one obtains

$$
F^{(d)}=\mathcal{L}_{\operatorname{lin}}[v]=\left[2 \frac{\partial \bar{u}}{\partial z} \nabla_{h}^{2} v+\left(\beta-2 \frac{\partial^{2} \bar{u}}{\partial y^{2}}\right) \frac{\partial v}{\partial z}\right]
$$

expressed entirely in terms of the perturbation meridional velocity $v ; F^{(d)}$ is referred to as the "dynamical forcing." The second term, $F^{(h)}=\nabla_{h}^{2} \dot{\Theta}$, depends explicitly on heating.

\section{d. Inverting the omega equation: Unconditional case}

By ignoring the conditional aspect of the parameterizations, $F^{(h)}$ becomes linear in $w$. It is then possible to express the vertical motion completely in terms of streamfunction by inverting the omega equation. Here, a Green function method is used for the inversion (e.g., Mak 1982, 1994). The situation without large-scale rain $\left(\epsilon_{\mathrm{LSR}}=0\right)$ is discussed below, and the extension with large-scale rain is derived $^{2}$ in appendix A.

The first step is to construct the Green function $\mathcal{G}_{w}$ for the operator $\mathcal{L}_{w}$ in (6), assuming a horizontally wavelike

\footnotetext{
${ }^{2}$ Alternatively, the unconditional LSR parameterization can be described using the "reduced- $N^{2}$ " approach, introduced by Eady (1949), where the $N^{2}$ is changed from its dry value to an effective $\tilde{N}^{2}(z) \equiv N^{2}-\epsilon_{\mathrm{LSR}} r(z)$.
}

perturbation. Because the vertical velocity vanishes at rigid lids, $\mathcal{G}_{w}$ satisfies simple homogeneous boundary conditions. By multiplying (6) from the left by $\mathcal{G}_{w}$ and integrating over the domain, one obtains

$$
w(z, t)=w^{(d)}(z, t)+w^{(h)}(z, t),
$$

where the rhs contains the two contributions

$$
\begin{aligned}
& w^{(d)}(z, t)=\int \mathcal{G}_{w}\left(z, z^{\prime}\right) F^{(d)}\left(z^{\prime}, t\right) d z^{\prime} \quad \text { and } \\
& w^{(h)}(z, t)=\epsilon_{\mathrm{CCV}} \alpha_{k}(z) w\left(z_{*}, t\right),
\end{aligned}
$$

where $\alpha_{k}(z) \equiv-\left(k^{2}+l^{2}\right) \int \mathcal{G}_{w}\left(z, z^{\prime}\right) h\left(z^{\prime}\right) d z^{\prime}$. The first contribution to $(8), w^{(d)}$, is referred to as the "dynamic" vertical velocity since it can be solved given $\psi$. The second, $w^{(h)}$, is only nonzero when $\epsilon_{\mathrm{CCV}} \neq 0$. It should be emphasized that in absence of baroclinic shear, moist normal modes can only exist if $w(z)=w^{(h)}(z)$, implying that $\epsilon_{\mathrm{CCV}} \alpha_{k}\left(z_{*}\right)=1$. This limit, which defines the socalled "CISK threshold" (Craig and Cho 1988), also enters the baroclinic problem. Equation (8) is solved by requiring it to be self-consistent at $z_{*}$, giving

$$
w\left(z_{*}, t\right)=\frac{w^{(d)}\left(z_{*}, t\right)}{1-\epsilon_{\mathrm{CCV}} \alpha_{k}\left(z_{*}\right)} .
$$

Since $w^{(d)}\left(z_{*}, t\right)$ is known from (9), using (11) one can obtain $w\left(z_{*}, t\right)$. Using $w\left(z_{*}, t\right)$ in $(10)$ gives $w^{(h)}(z, t)$, which upon substitution into (8) gives $w(z, t)$. It is clear from (11) that $w^{(h)}\left(z_{*}, t\right)$ [and therefore $w\left(z_{*}, t\right)$ ] becomes singular at the CISK threshold $\epsilon_{\mathrm{CCV}} \alpha_{k}\left(z_{*}\right)=1$ unless $w^{(d)}\left(z_{*}, t\right)=0$. The singular behavior near the CISK threshold is an intrinsic aspect of moisture parameterizations based on the total vertical velocity. It typically occurs at a particular $\left(k, \epsilon_{\mathrm{CCV}}\right)$ combination and is the basic result of a positive feedback between heating and vertical velocity. Although it might be relevant for the zero-shear situation, we will use the CISK threshold mainly to determine an upper bound on the heating intensity. The maximum allowed heating intensity will be chosen such that $\epsilon_{\mathrm{CCV}} \alpha_{k}\left(z_{*}\right)<1$ for all wavenumbers. ${ }^{3}$

\section{e. Model basis formulated using kernel Rossby waves}

For a single zonal wavenumber $k$, the differential operator $\mathcal{L}_{\psi}$, which relates streamfunction to $\mathrm{PV}$ via $\mathcal{L}_{\psi}[\psi]=$ $q$, can be inverted by means of a Green function $\mathcal{G}_{\psi}$, such

\footnotetext{
${ }^{3}$ Another way to prevent the formation of the singularity through the heating vertical velocity feedback, is to use only the "dynamic" vertical velocity $w^{(d)}\left(z_{*}, t\right)$ [rather than $\left.w\left(z_{*}, t\right)\right]$ in the CCV parameterization (Mak 1982).
} 
that $\psi(z)=\int \mathcal{G}_{\psi}\left(z, z^{\prime}\right) q\left(z^{\prime}\right) d z^{\prime}$. By using $\partial \mathcal{G}_{\psi} / \partial z=0$ at rigid lids, and $\mathcal{G}_{\psi} \rightarrow 0$ at infinity if no upper boundary is present, $\mathcal{G}_{\psi}\left(z, z^{\prime}\right)$ represents the streamfunction that can be associated with a PV $\delta$ function at $z=z^{\prime}$ and zero potential temperature at rigid lids. Such a structure was called a kernel Rossby wave (KRW) by Heifetz and Methven (2005). Using $\mathcal{G}_{\psi}$, the PV equation can be written as

$$
\frac{\partial q}{\partial t}+i k \mathcal{A}[q]=S
$$

where $\mathcal{A}[q]$ is a linear integral operator acting on the PV state $q$ (e.g., V09), and $S$ represents diabatic processes acting on wave $k$. In formulating (12), the Bretherton (1966) approach is used to interpret boundary potential temperature contributions in terms of boundary PV $\delta$ functions. If the parameterizations are taken in their unconditional form, $S$ can be expressed entirely in terms of $\mathrm{PV}$, implying that (12) can be written more compactly as

$$
\frac{\partial q}{\partial t}+i k \tilde{\mathcal{A}}[q]=0 .
$$

Appendix B gives a rigorous derivation of (13), including the definition of $\tilde{\mathcal{A}}$, on the basis of KRW theory. The problem has been reduced from a set of coupled partial differential equations (PV equation and omega equation) to a single linear ordinary differential equation for the PV.

\section{Two simple examples}

This section describes two examples, where (13) assumes a particularly simple form yet retains the primary effects of moisture. The first is wave-CISK instability for a constant-shear flow in a vertically unbounded domain, previously discussed by SL91. In the second a lower boundary is added. The main focus will be on the normal modes for which a classification is proposed in section $3 \mathrm{c}$.

\section{a. Vertically unbounded shear flow with cumulus heating (f plane)}

A constant-shear flow in a vertically unbounded domain on the $f$ plane is the simplest geometry supporting gNMs in the presence of a wave-CISK moisture parameterization. Since $\bar{q}_{y}=0$ throughout the domain, there can be no dry baroclinic instability. If the heating profile takes the form of a top-hat, diabatically induced PV growth can only occur at the two edges of the heating profile. The normal-mode analysis of this system is given by SL91, who used a different route-avoiding the omega equation - to obtain the normal-mode results. Here we focus on aspects that either have not been addressed by SL91 or that are important to later sections, when more complicated geometries are introduced. Following SL91, the heating intensity $\epsilon_{\mathrm{CCV}}$ is made proportional to the "cloud depth" $\Delta z$ (depth of the heating layer)

$$
\epsilon_{\mathrm{CCV}}=E \Delta z
$$

where the parameter $E=O(1)$ is called the "diabatic heating efficiency." The system has two intrinsic vertical scales: the Rossby height scale $H_{R} \equiv 1 / K$ (in units of $H$ ), where $K=\left(k^{2}+l^{2}\right)^{1 / 2}$, and $\Delta z=z_{2}-z_{1}$, the depth of the heating layer. The normal-mode dispersion relation is a function of $k \Delta z$ and the diabatic heating efficiency $E$ only. To avoid reaching the CISK threshold for any $k$, we impose the constraint $E<2$.

With the previous approximations (13) reduces to a twowave problem for the PV waves at the bottom (labeled 1) and top (labeled 2) of the heating profile. The total PV can be written as $q(z, t)=q_{1}(z, t)+q_{2}(z, t)=a_{1}(t) \delta\left(z-z_{1}\right)+$ $a_{2}(t) \delta\left(z-z_{2}\right)$, where $a_{1,2}(t)=A_{1,2}(t) \exp \left[i \epsilon_{1,2}(t)\right]$ and $A_{i}$ and $\epsilon_{i}$ denote the amplitude and phase of the PV and circulation anomalies associated with the two CRWs. The time evolution of $a_{i}$ is given by (H04; V09)

$$
\dot{a}_{i}(t)=-i k \sum_{j} c_{i j} a_{j}(t), \quad i, j \in(1,2),
$$

where $c_{i j}=\bar{u}\left(z_{i}\right) \delta_{i j}-\gamma_{i j} / k$. The interaction coefficients $\gamma_{i j}=\gamma\left(z_{i}, z_{j}\right)$ are computed in (B7) [note that $\gamma^{(d)}\left(z_{i}, z_{j}\right)=$ 0 in this case]. The expressions for $c_{i j}$ show that the propagation and interaction of the PV $\delta$ functions (KRWs), which exist on the edges of the heating layer, is very similar to the propagation of KRWs on a dry PV gradient. In the dry case, the sign of $\bar{q}_{y}$ determines the propagation direction, and the actual propagation speed is given by the product of $\bar{q}_{y}$ and the $\mathrm{PV}$-induced local meridional wind. Since the meridional velocity of a KRW is in quadrature with its PV, isolated KRWs are neutral, and unstable waves can only form if there are at least two KRWs. A similar situation occurs in the moist case. Ascent induces a positive $\mathrm{PV}$ tendency in regions where there is a positive gradient in heating, $h_{z}>0$ (at the lower edge of the heating profile), and a negative PV tendency where $h_{z}<0$ (at the top edge). Because the vertical velocity associated with a KRW is also in quadrature with its $\mathrm{PV}$, the diabatic processes are able to propagate an existing PV pattern relative to the flow, with the sign of $h_{z}$ determining the propagation direction. The actual propagation speed is given by the product of $h_{z}$ and the vertical velocity induced at $z_{*}$.

There are two necessary conditions for instability to occur. In terms of the notation of (15), these conditions are (i) $\gamma_{i j} \gamma_{j i}<0$ and (ii) $\bar{u}_{i} \gamma_{i j}>0$. In physical terms, these conditions require that the waves must propagate in 

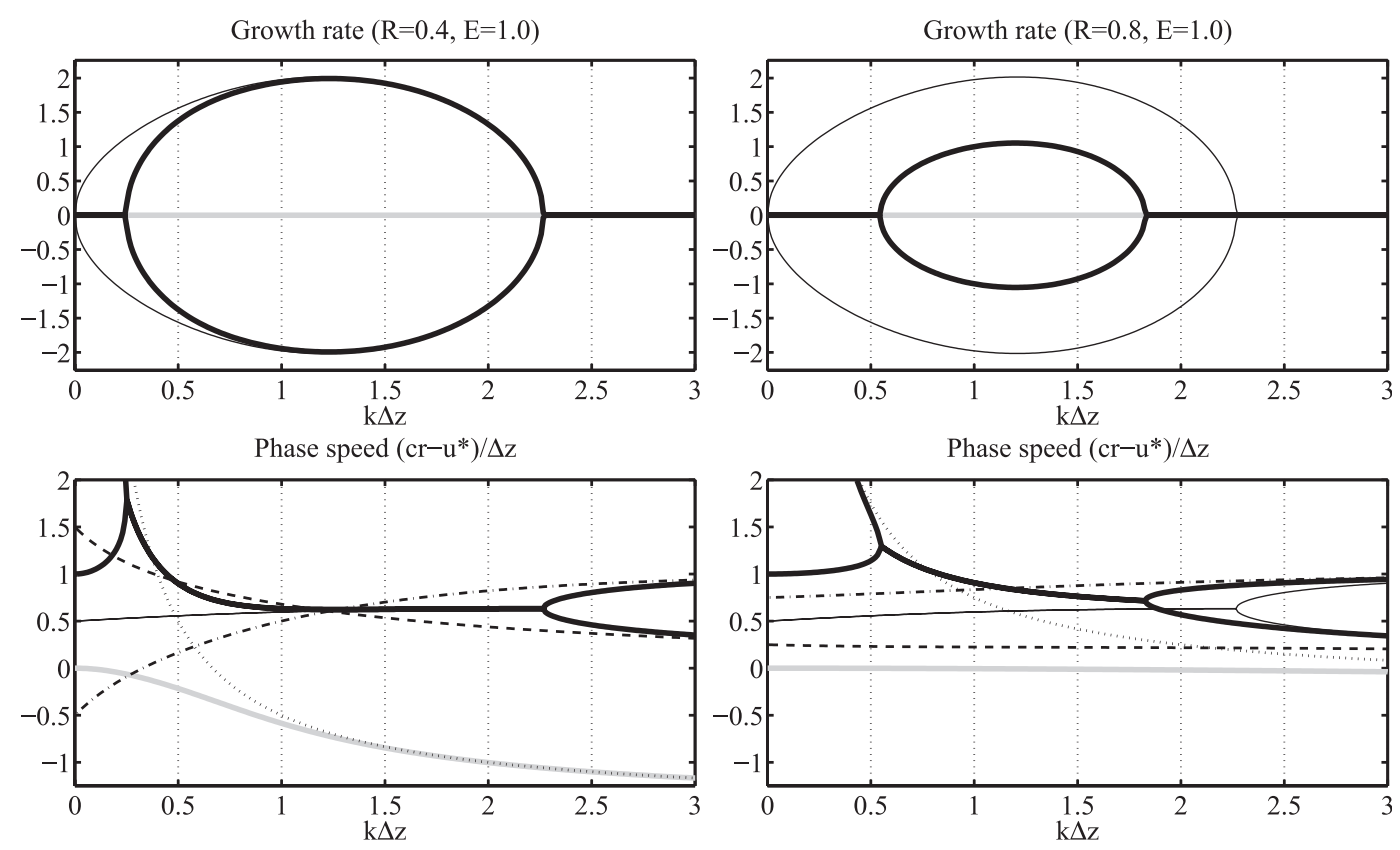

FIG. 1. Dispersion relations for the unbounded and semi-infinite constant-shear flow with cumulus heating. (top) Growth rate (scaled by max dry Eady growth rate); (bottom) scaled phase speed $\left[c_{r}-\bar{u}(z *)\right] / \Delta z$. Thin full lines show the unbounded SL91 problem; the heavy black/gray curves show the unstable/neutral normal modes of the semiinfinite problem. Black lines in the bottom panels show the propagation rates $\left[c_{i i}-\bar{u}(z *)\right] / \Delta z$ of the three CRW components ( $c_{B B}$ dotted, $c_{11}$ dashed, and $c_{22}$ dash-dotted). Heating parameter values, defined by (14) and (16), are (left) $E=1, R=0.4$ and (right) $E=1, R=0.8$.

opposite directions to one another and counter to the shear in the zonal flow, respectively. This is true both for the dry and the moist problem and agrees with Hayashi and Young (1987).

\section{b. Semi-infinite Eady model with cumulus heating (f plane)}

Because of the absence of a lower boundary, the problem studied in the previous section is oversimplified as a model for moist atmospheric cyclogenesis. In the second example the lower boundary is added at $z_{b}=0$. Other conditions are unchanged.

The main difference between the unbounded and the semiunbounded domain is that the latter supports potential temperature edge waves, which propagate on the surface mean meridional temperature gradient. These boundary potential temperature perturbations are interpreted as boundary PV anomalies, which propagate relative to the mean flow on the negative surface mean PV gradient (Bretherton 1966). Thus, there are now three spatially distinct PV waves that define three RW components: $q_{B}$ (the surface edge wave), and $q_{1,2}$ (the PV waves at the lower edge and the top edge of the heating profile). With three PV components, there exist three possible pairings, namely $\left(q_{B}, q_{1}\right),\left(q_{B}, q_{2}\right)$, and $\left(q_{1}, q_{2}\right)$. Instability and sustained mutual growth, however, can only occur between components of two of the three pairs that can be formed, since the $\left(q_{B}, q_{1}\right)$ pair does not satisfy the conditions for mutual amplification for heating intensities below the CISK threshold.

With the lower boundary included, a new dynamically important height scale appears: the distance between the top of the heating layer and the surface. This motivates the introduction of the ratio of height scales $R$ :

$$
R=\frac{\Delta z}{z_{2}-z_{b}}
$$

For given values of $E$ and $0 \leq R \leq 1$, the normal-mode growth rate is a function of $k \Delta z$. Realistic values of $R$ can be obtained from estimates of $z_{1}-z_{b} \in[0.1,0.3]$ (in units of $H$ ) and $z_{2}-z_{b} \in[0.5,0.9]$, giving $R \in[0.4,0.9]$. To remain below the CISK threshold, a further constraint is that $E<2$ (at least if $z_{*}=z_{1}$ ).

Figure 1 shows the normal-mode dispersion relation for two characteristic cases in which $E=1$. For each wavenumber there are three normal modes, two of which possibly form a growing-decaying conjugate pair. The third mode (gray lines) remains neutral over the entire $k \Delta z$ domain and only for this mode $q_{2}$ plays a negligible role. At large $k \Delta z$ it resembles the isolated boundary edge wave, whereas $q_{B} \sim-q_{1}$ in the limit $k \Delta z \rightarrow 0$. The attention will be on the other two modes. Comparing the 
dispersion relation to the unbounded problem, one immediately notices that the maximum growth rate is reduced because of the presence of the lower boundary, and further that the maximum growth rate decreases with $R$. For $R \neq 0$ a longwave cutoff wavenumber appears and, as expected, no growth remains in the limit $R \rightarrow 1$ (when $z_{1} \rightarrow z_{b}$, or $z_{2} \rightarrow \infty$ ). Interestingly, the longwave cutoff is never far off $k \Delta z \sim R$, or, in other words, the Rossby height scale $H_{R}=1 / K \sim\left(z_{2}-z_{b}\right)$.

The results imply, perhaps counterintuitively, that the lower boundary acts to suppress rather than to invigorate the baroclinic instability, and that this suppression occurs increasingly at longer zonal wavelengths. The reason is that the wave-CISK parameterization cannot operate efficiently if $z_{*}$ is near the surface ["near the surface" here means $k\left(z_{*}-z_{b}\right) \ll 1$ ], because it is difficult to generate sufficient vertical motion at such small distances above $z_{b}$. This becomes even more clear when looking at the self-interactions of the PV waves above and below the heating, $c_{11}$ and $c_{22}$, for $R=0.8$ (bottom right panel in Fig. 1, dashed and dash-dotted black lines, respectively), which hardly vary with $k \Delta z$, their values being roughly predicted by the mean zonal wind at their home base.

\section{c. Classification of growth mechanisms}

Only two out of three possible pairings of CRWs may produce sustained growth in the case of the semi-infinite wave-CISK Eady model $\left[\left(q_{2}, q_{1}\right)\right.$ and $\left.\left(q_{2}, q_{B}\right)\right]$, suggesting that its growing normal modes fall into two classes. It is easy to determine to which "regime" a particular gNM belongs, since for a fixed set of parameters the equations describing the evolution of the three CRW components $\left(q_{B}, q_{1}, q_{2}\right)$ are identical to (15), except that the system is now $3 \times 3$. The growth rate of each RW component follows from the real part of (15):

$$
\frac{d}{d t} \ln A_{i}=-k \sum_{j} c_{i j} \frac{A_{j}}{A_{i}} \sin \left(\epsilon_{i}-\epsilon_{j}\right),
$$

where now $(i, j) \in(B, 1,2)$. Equation (17) is used to determine which of the two eastward-propagating components $\left(q_{B}\right.$ or $\left.q_{1}\right)$ contributes the most to the growth rate of the westward-propagating component $\left(q_{2}\right)$, yielding two classes of unstable normal modes:

1) Q2-Q1: $q_{2}$ amplifies mostly due to $q_{1}$ and vice versa. This is the type of instability discussed in SL91 in the limit of strong heating far from boundaries.

2) Q2-QB: $q_{2}$ amplifies mostly due to $q_{B}$ and vice versa. This will be referred to as a diabatic Rossby wave (DRW) since its existence depends on both nearsurface baroclinicity and latent heating.

This classification can be extended in a natural way if an upper boundary and interior mean PV gradient are
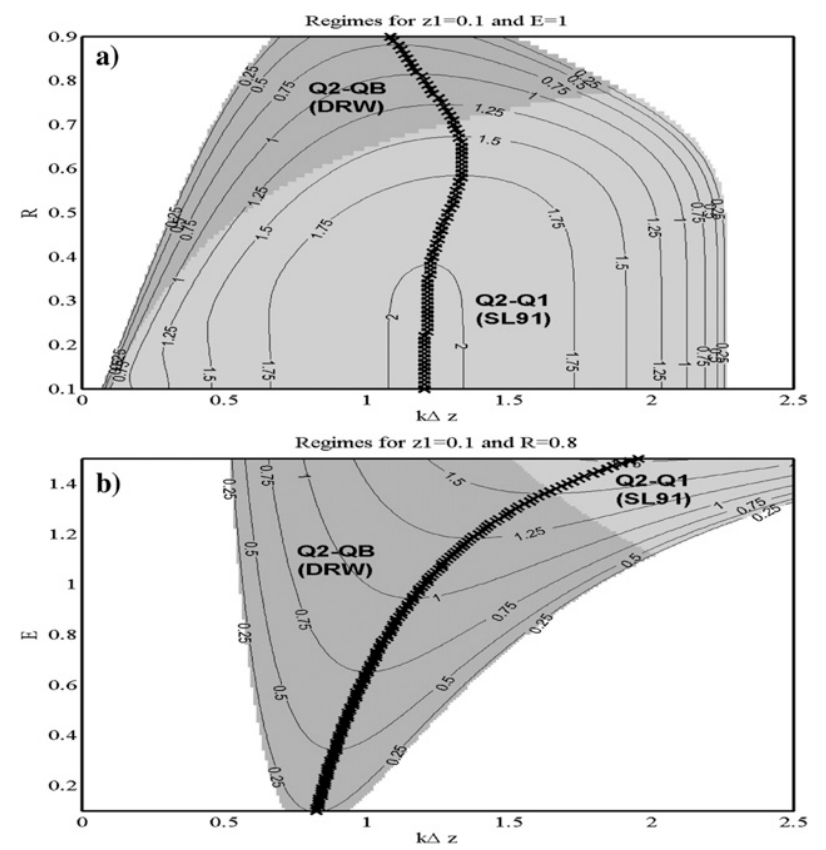

FIG. 2. Classification diagram for the semi-infinite Eady model with wave-CISK parameterization. Also shown are contours of normal-mode growth rate (scaled by the dry maximum Eady growth rate). (top) Fixed $E=1$ [Eq. (14)] and varying $k \Delta z$ and $R$ [Eq. (16)]. (bottom) Fixed $R=0.8$, and varying $k \Delta z$ and $E$. The black crosses mark the location of the most unstable wave for each value on the vertical axis.

present (next sections). Please note that in the classification, the amplitude ratios $A_{i} / A_{j}$ and phase differences $\epsilon_{j}-\epsilon_{i}$ assume the values for a specific normal mode and are not free parameters.

Figure 2 shows the normal-mode classification diagram for a situation in which either $E$ or $R$ is fixed, and $R$ or $E$ is varied along with $k \Delta z$. The dark gray shading highlights normal modes of type Q2-QB (DRW) and the light-gray area those of type Q2-Q1. (White areas indicate zero normal mode growth rate). The first observation is that both regimes indeed occur. The Q2-Q1 regime is largely confined to large $E$ values and small $R$ values (i.e., efficient heating far from boundaries), whereas the Q2-QB regime is confined to longer wavelengths (small $k \Delta z$ ), large $R$ (deep heating close to ground), and small $E$ (heating efficiency). Note that there are no regions of overlap possible following the regime definition. The black crosses mark the $k \Delta z$ value of the fastest-growing normal mode for a given value of the $y$ ordinate. Interestingly, fastestgrowing normal modes can be found of both types.

For a range of points in the $E-R$ plane the fastestgrowing normal mode was found, and its type analyzed. This produced the classification diagram for the fastestgrowing normal mode, shown in Fig. 3. In the lowerright half of the $E-R$ plane the most unstable waves are 


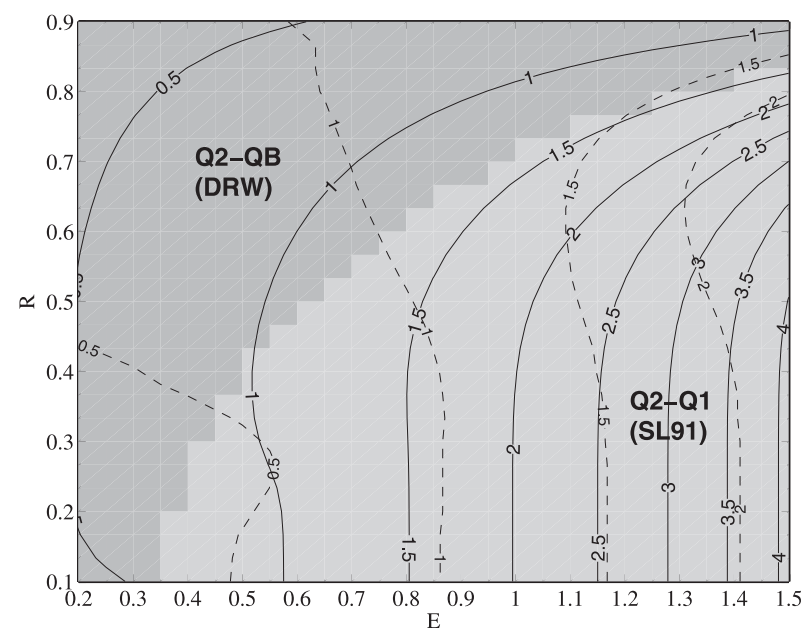

FIG. 3. $E-R$-plane classification diagram for the most unstable wave of the semi-infinite Eady model with wave-CISK parameterization. Also shown are the growth rate (full contours, scaled by Eady max growth rate) and $k \Delta z$ of the wave (dashed contours).

of the Q2-Q1 type, whereas for small values of $E$ and larger values of $R$, they resemble the DRW. The transition between the different regimes is smooth, implying that in the transition zone the modes are of a mixed type, involving significant interaction between all three CRWs.

\section{Eady model with cumulus heating}

Continuing the progression of increasing model complexity, the next step is to add a tropopause in the form of a rigid lid. If $S=0$ the model is the classic Eady (1949) model. Mak $(1982,1994)$ studied the Eady model with cumulus heating. Since the Eady model supports dry baroclinic instability, one of the questions is how the normalmode classification changes.

The upper lid introduces an additional Rossby edge wave $q_{T}$ propagating along the positive PV gradient there. Based on the possible pairings between the four CRW components $\left(q_{B}, q_{1}, q_{2}, q_{T}\right.$; waves on the ground, lower and upper edges of the heating, and the tropopause, respectively) that may lead to sustained modal growth, two new regimes are defined:

3) QT-QB: $q_{T}$ amplifies mostly due to $q_{B}$ and vice versa. Also referred to as $d r y$ growth.

4) QT-Q1: $q_{T}$ amplifies mostly due to $q_{1}$ and vice versa. Also referred to as type- $C$ growth because of the interaction between tropopause troughs and the heating layer (Plant et al. 2003).

The regime classification algorithm is as before and uses the $4 \times 4$ equivalent of (15) and (17). Obviously, with the
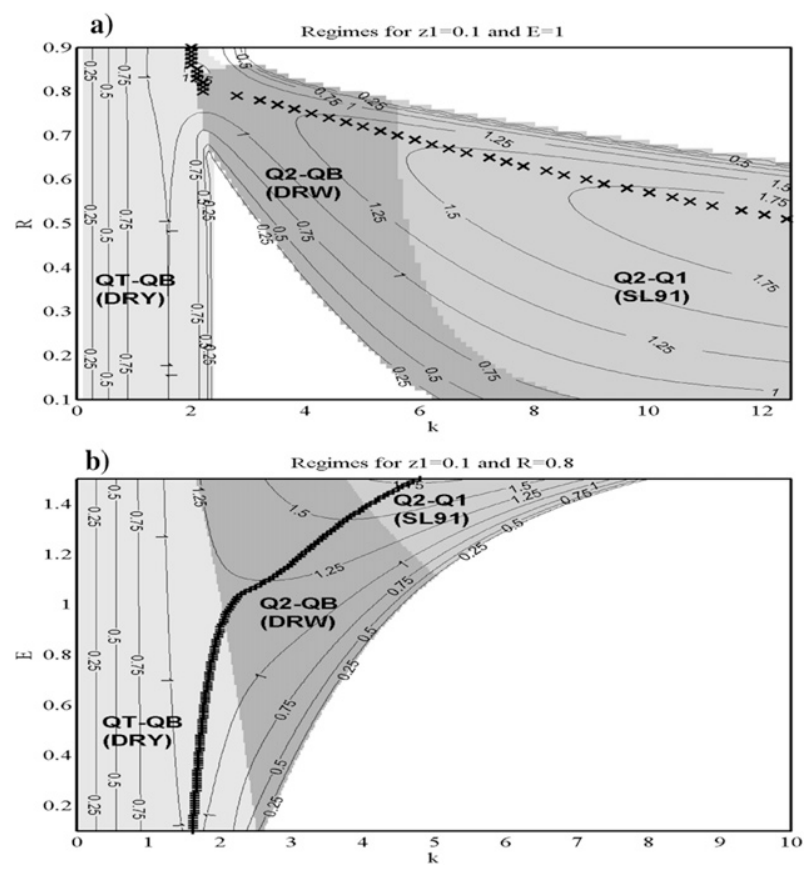

FIG. 4. Classification diagrams for the wave-CISK Eady model. Also shown are contours of normal-mode growth rate (scaled by the dry maximum Eady growth rate). (a) Fixed $E=1$, and varying $k$ and $R$; (b) fixed $R=0.8$, and varying $k$ and $E$. The black crosses mark the location of the most unstable wave for each value on the vertical axis.

introduction of the tropopause, the tropopause height appears as a new dynamically important height scale. As a result, the dispersion relation is no longer a function of $E, R$, and $k \Delta z$ only. The strategy chosen for the parameter sensitivity study is to set $z_{t}-z_{b}=H$ and $z_{1}-z_{b}=$ $0.1 H$ and to vary the cloud depth $\Delta z=z_{2}-z_{1}$ and $E$ ( $H$ is the height scale from section $2 a$ ). The height of the moist layer $z_{*}$ is kept constant, although changing its location will have some effect (Mak 1994).

Figure 4 shows the normal-mode classification diagram for cases similar to those discussed for the semiunbounded domain. Note that the $x$ ordinate is $k$, rather than $k \Delta z$ in Fig. 2. The reason for using $k$ is that in this way the dry Eady shortwave cutoff at $k \sim 2.4$ becomes very obvious, and furthermore that all longwave modes with $k \leq 2.4$ classify as QT-QB (DRY), with diabatic heating playing an insignificant role for all $R$. For small $R$ values these DRY modes are separated from the modes in which moisture plays a more important role, by a clear "gap" indicated by the white region in which no modal instability is found. At the shortwave end, the figures look very similar to those of Fig. 2 (e.g., moist shortwave cutoff at $k \Delta z \sim 2.2$ for small $R$ ).

The QT-Q1 regime is not identified for the current set of parameters. However, it is clear that because of the 

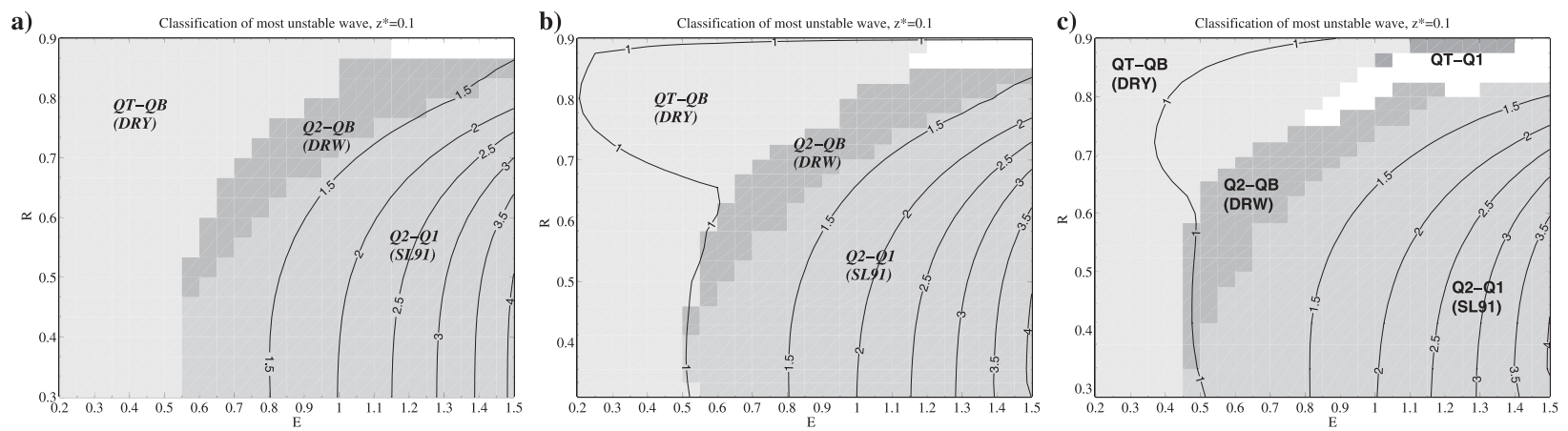

FIG. 5. $E-R$-plane classification diagram for the most unstable wave for various wave-CISK models with a top-hat heating profile and $z_{*}=z_{1}=0.1$ : (a) Eady model, (b) Green model, and (c) Charney model ( $\beta$ was increased to give the same wavelength of the most unstable dry wave as in the Eady model). Also shown is the growth rate (full contours, scaled by Eady max growth rate).

symmetry of the Eady model, the area covered by Q2-QB would be covered by QT-Q1 if instead of $\left(z_{1}=a, z_{2}=b\right.$, $\left.z_{*}=z_{1}\right)$ the model setup had been $\left(z_{1}=z_{t}-b, z_{2}=z_{t}-a\right.$, $z_{*}=z_{2}$ ). However, it is unlikely that physically realistic values of $z_{*}$ are near the top of the cloud. Therefore, despite the fact that $z_{*}$ is much closer to the bottom of the heating layer $z_{1}$ than to the top $z_{2}$ the PV wave at the top of the cloud $Q_{2}$ is more important for baroclinic growth than $Q_{1}$. Of course, this picture would change if the bottom of the cloud $z_{1}$ and $z_{*}$ were located much higher (e.g., in the midtroposphere), with a cloud top at tropopause level. But again, these are not considered to be the most realistic cases.

Figure 5 a gives the classification diagram in the $E-R$ plane for the most unstable waves. As expected a significant part of the left half of the $E-R$ plane is covered by the QT-QB, or DRY regime. Note further that there is no overlap between the regimes in this figure, although this is not obvious-for instance, according to our simple classification algorithm, a gNM could in theory qualify both as Q2-Q1 or as QT-QB (if for both pairs these components have largest mutual amplification rates), since they describe interactions between different pairs (similarly for Q2-QB and QT-Q1). As in the previous figure, fastestgrowing normal modes dominated by type-C interaction are not found in this figure. The transition between Q2-Q1 and QT-QB is discontinuous rather than smooth.

\section{More general basic states including interior PV gradients}

In the previous sections we have considered flows for which there was a clear spatial separation between the PV anomalies that played a role in growing normal modes. As a result, CRW components were readily identified and analyzed. H04 have shown that such a partitioning into CRW components is also possible for the dry problem in the more general situation when the interior PV gradient is nonzero throughout the domain. In this section the theory is extended to the moist initial value problem following the approach of V09.

\section{a. On the origin of $P V$ perturbations}

In the Eady model with a top-hat cumulus heating parameterization, two of the CRWs (i.e., of the surface and tropopause edge waves) that appear in the gNM can be related to meridional displacements $\eta$ of the basic-state PV contours. Following V09, this displacement $P V$ is denoted by

$$
q^{(d)}=-\eta \frac{\partial \bar{q}}{\partial y}
$$

In the same way, the PV associated with the remaining two RW components can be related to the diabatic heating and is denoted by $q^{(m)}$. Note that the CRWs are always labeled with a subscript (e.g., $q_{B}, q_{1}$ ) and that PV of different origin is denoted with a superscript [e.g., $q^{(m)}$. By substituting the definition of $q^{(d)}$ into (1), and using the kinematic relation $\partial \eta / \partial t+\bar{u} \partial \eta / \partial x=v$, the following evolution equations are obtained:

$$
\begin{aligned}
& \frac{\partial q^{(d)}}{\partial t}+\bar{u} \frac{\partial q^{(d)}}{\partial x}=-v \frac{\partial \bar{q}}{\partial y}, \\
& \frac{\partial q^{(m)}}{\partial t}+\bar{u} \frac{\partial q^{(m)}}{\partial x}=S, \quad \text { and } \\
& \frac{\partial q^{(p)}}{\partial t}+\bar{u} \frac{\partial q^{(p)}}{\partial x}=0 .
\end{aligned}
$$

In general, initial conditions include PV that cannot be related to meridional displacements or heating. Equation (21) shows that this remaining PV is simply advected by the basic-state zonal flow as if passive. These equations are coupled through $v$ and $S$, which depend on all three PV components.

The above partitioning can be readily applied in the more general situation where distributed heating occurs 

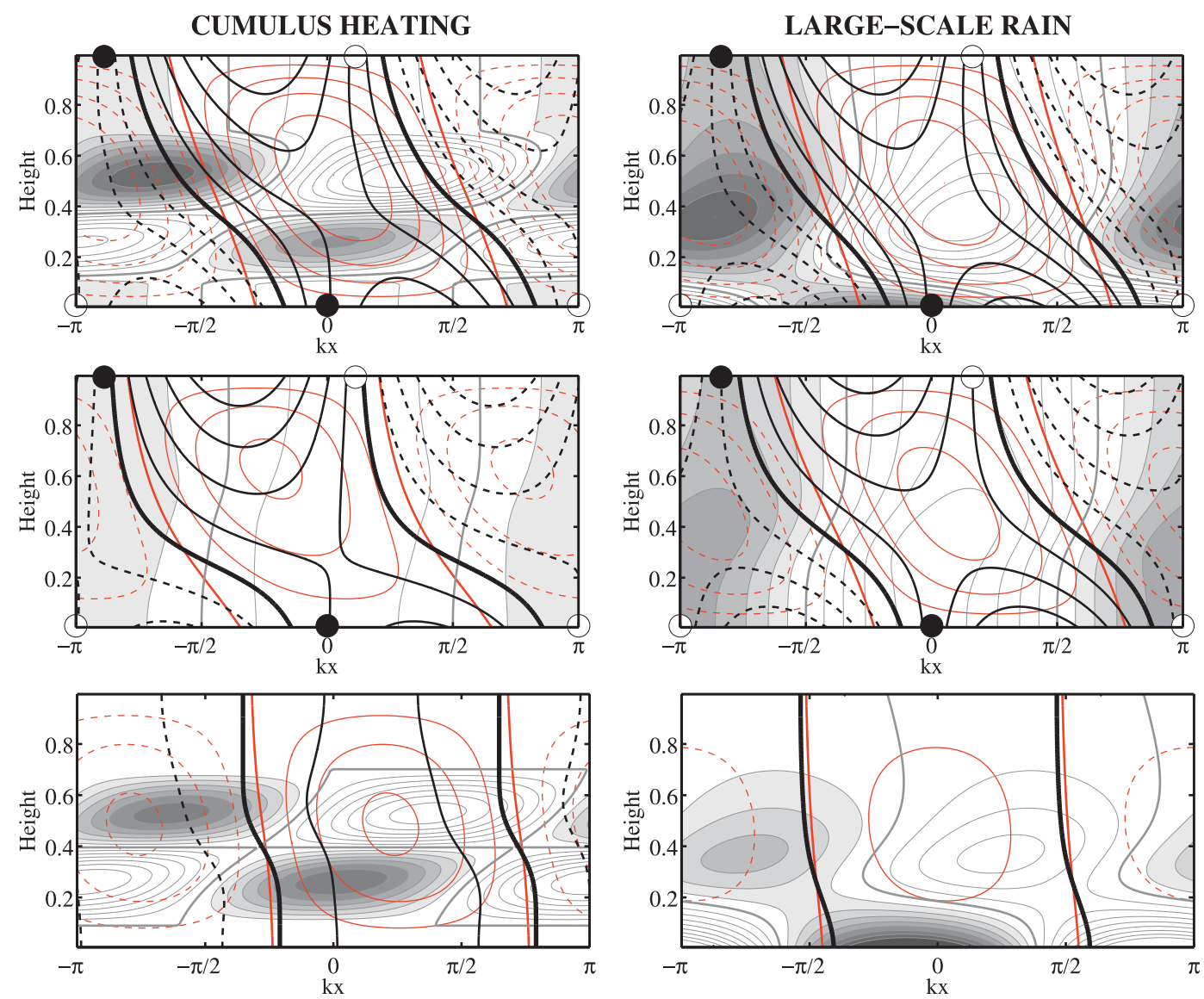

FIG. 6. Decomposition of the gNM $q_{G}=q_{G}^{(d)}+q_{G}^{(m)}$ for a wave with $k=1.6$ (dry Eady maximum) growing on the Green model basic state (with rigid lid and $\beta \neq 0$ ). (left) Case with cumulus heating $\left(E=1, z_{1}=0.1, z_{2}=0.7\right.$ ); (right) Large-scale rain $\left[\epsilon_{\mathrm{LSR}}=0.9\right.$ and $\left.r=\exp (-z / 0.3)\right]$; (top to bottom): full $q$, displacement $q^{(d)}$, and moist $q^{(m)}$. Displayed are PV (shading, positive is dark), boundary PV (circles, positive is dark), meridional wind (black contours; negative values are dashed) and vertical wind (red contours; negative values are dashed). The cumulus heating case has slightly larger growth rate $(0.36$ vs 0.33$)$.

and a nonzero interior PV gradient is present. However, whenever there is spatial overlap between the regions where $\bar{q}_{y} \neq 0$ and $S \neq 0$, there may be ambiguity as to how to partition a general PV state vector $q$ into $q^{(d)}$, $q^{(m)}$, and $q^{(p)}$, at least as long as one does not know the exact history of the air. In contrast, for a NM with $q=$ $\operatorname{Re}\left\{q_{G}(z) \exp [i k(x-c t)]\right\}$, the partitioning is given by

$$
q_{G}^{(d)}=\frac{-\bar{q}_{y} v_{G}}{i k(\bar{u}-c)}, \quad q_{G}^{(m)}=\frac{S_{G}}{i k(\bar{u}-c)}, \quad q_{G}^{(p)}=0,
$$

where $v_{G}$ and $S_{G}$ are the meridional wind and diabatic PV term of the gNM, respectively. These expressions are nonsingular for growing (or decaying) normal modes with complex phase speed $c$.

For example, in the Eady model with top-hat heating $q^{(d)}$ consists of boundary PV only and $q^{(m)}$ consists of interior $\mathrm{PV}$ at the two heating edges only. In the Eady model with large-scale rain, $q^{(d)}$ exists only at the boundaries but $q^{(m)}$ is distributed everywhere in the interior. In the Charney model with top-hat heating, $q^{(d)}$ is distributed everywhere but $q^{(m)}$ exists at only two levels. Two further examples are shown in Fig. 6. Note the similarity in modal structures for the CCV and LSR parameterizations, especially the displacement $\mathrm{PV}$ and associated winds.

\section{b. Definition of general $R W$ components}

In this section RW components are defined as suitable superpositions of $q_{G}^{(d)}$ and its complex conjugate and of $q_{G}^{(m)}$ and its complex conjugate. We start with a brief summary of the dry theory. In absence of diabatic heating $(S=0)$, the gNM must be associated exclusively with displacement PV $q_{G}^{(d)}$. In that case a framework exists to analyze baroclinic instability in terms of two interacting RW components, known as CRWs (H04). Central to CRW theory is that the dry gNM and its decaying complex conjugate (dNM) can be suitably rewritten as a sum 
of two vertically untilted RW components called CRWs. Because CRWs have vertically untilted PV structure, their associated wind field is also vertically untilted. As a result, the CRWs are neutral and self-propagating in isolation but will excite each other in time and may eventually phase-lock in a mutually amplifying configuration.

In the dry theory, the CRWs can be unambiguously defined from the gNM: they are constructed to be orthogonal with respect to pseudomomentum and with respect to the wind-weighted term in the expression for pseudoenergy (H04). Denoting the CRWs' PV structures by $q_{B, T}$, the orthogonality conditions are

$$
\left\{q_{B}, \mathcal{N}_{d} q_{T}\right\}=0, \quad\left\{q_{B}, \mathcal{N}_{d} \bar{u} q_{T}\right\}=0,
$$

where the brackets define the inner product $\{X, Y\}$ $\int X^{*} Y \bar{\rho} d z$ and $\mathcal{N}_{d}=\left(\bar{q}_{y}\right)^{-1}$ is the weighting. The same technique is used to obtain two "dry CRWs", $q_{B, T}$, from $q_{G}^{(d)}$ and its complex conjugate in case $S \neq 0$; so called because in the limit $S \rightarrow 0$ they become the CRWs of the dry theory.

Two further moist CRWs (labeled $q_{1,2}$ ) are derived from the moist PV associated with the $\mathrm{gNM}, q_{G}^{(m)}$, and from its complex conjugate. Similar to the dry CRWs, the moist CRWs are also defined to be orthogonal with respect to two global integrals,

$$
\left\{q_{1}, \mathcal{N}_{m} q_{2}\right\}=0, \quad\left\{q_{1}, \mathcal{N}_{m} \bar{u} q_{2}\right\}=0 .
$$

The weighting $\mathcal{N}_{m}$ for simplicity is taken to be equal to $\mathcal{N}_{d}$.

Some comments on the particular choice of $\mathcal{N}_{d, m}$ are now given. Held (1985) showed that the discrete normal modes of the dry problem are orthogonal to all continuous spectrum modes $(\mathrm{CMs})$ in the pseudomomentum inner product, $\left\{q_{i}, \mathcal{N}_{d} q_{j}\right\}=\delta_{i j}$. Therefore, by making the CRWs of the dry theory also orthogonal in this inner product, the CRWs and CMs form a complete and orthogonal basis. In the case with moisture parameterizations included, dry pseudomomentum is no longer a conserved quantity, implying that the modes are no longer orthogonal under the pseudomomentum inner product. Although one can easily demonstrate that the moist normal modes are orthogonal with respect to some matrix norm, ${ }^{4}$ the authors have not been able to express this

\footnotetext{
${ }^{4}$ The vertically discretized normal-mode eigenvalue problem is of the form $\mathbf{A} y=c y$. Since $\mathbf{A}$ in the problems considered is both real and nonsingular, it is always possible to "symmetrize" the eigenvalue problem with a symmetrizer $\mathbf{X}$ ( $\mathbf{X}$ is real symmetric) that satisfies $\mathbf{X A}=(\mathbf{X A})^{\dagger}$ (e.g., Sen and Venkaiah 1988). By writing $\mathbf{X}=\mathbf{P}^{\mathrm{T}} \mathbf{D P}$ the eigenvalue problem becomes $\mathbf{B} x=c \mathbf{D} x$, where $\mathbf{B}=\mathbf{P X A P}^{\mathrm{T}}, x=\mathbf{P} y$, and $\mathbf{D}$ is a diagonal matrix. It can be shown that neutral modes are orthogonal in the sense that $\left(c_{2}-c_{1}^{*}\right)\left\{x_{1}, \mathbf{D} x_{2}\right\}=0$.
}

norm in quadratic physical quantities. The one exception is the case where there is cumulus heating only, $\bar{q}_{y}=0$ and there are no boundaries. In that limit, the moist normal modes can be shown to be orthogonal with respect to the inner product $\left\{q_{i}, \mathcal{N}_{m} q_{j}\right\}=\delta_{i j}$, where $\mathcal{N}_{m}$ is proportional to ${ }^{5}(\partial h / \partial z)^{-1}$.

\section{c. Evolution equations for general RW components}

For the moment it is assumed that the PV state vector can be expressed entirely in terms of linear combinations of the gNM and dNM. By projecting (19) and (20) onto the four RW components using the brackets, one obtains [cf. (15)]

$$
\dot{a}_{i}(t)=-i k \sum_{j} c_{i j} a_{j}(t), \quad i, j \in(B, 1,2, T),
$$

where $a_{j}=A_{j}(t) \exp \left[i \epsilon_{j}(t)\right]$ denotes the complex amplitude of $\mathrm{CRW}-j$, and $c_{i j}$ are the propagation and interaction coefficients,

$$
c_{i j}=\tilde{u}_{i} \delta_{i j}-\tilde{\gamma}_{i j} / k
$$

where

$$
\tilde{u}_{i}=\frac{\left\{q_{i}, \mathcal{N} \bar{u} q_{i}\right\}}{\left\{q_{i}, \mathcal{N} q_{i}\right\}}, \quad \tilde{\gamma}_{i j}=\frac{\left\{q_{i}, \mathcal{N} \bar{\gamma} q_{j}\right\}}{\left\{q_{i}, \mathcal{N} q_{i}\right\}},
$$

with $\mathcal{N}=\mathcal{N}_{d}$ and $\bar{\gamma}=\gamma^{(d)}$ for $i \in(B, T)$, and $\mathcal{N}=\mathcal{N}_{m}$ and $\bar{\gamma}=\gamma^{(m)}$ for $i \in(1,2)$ (see appendix B). A key difference between (15) and (24) is that (24) describes the evolution of amplitude and phase of general RW components that have possibly highly intricate, vertically overlapping PV structures, whereas (15) describes the evolution of spatially separated $\mathrm{PV} \delta$ functions that formed the RW components in the wave-CISK Eady model. However, the interaction mechanisms are still basically the same. Therefore, the same normal-mode classification algorithm can be applied as in the Eady model with cumulus heating, where the general RW components replace the PV $\delta$ functions [cf. (17)]. Results for the Green (1960) model (Eady model with nonzero $\beta$ ) and the Charney (1947) model with cumulus heating are shown in Fig. 5. Note how similar these figures are, while the structure of the dry CRWs is significantly different in the models [see, e.g., Methven and De Vries (2008) for the structure of the CRWs in the Green model].

\footnotetext{
${ }^{5}$ In this case $\mathcal{N}_{m}^{-1}=\epsilon_{\mathrm{CCV}} \bar{\rho}^{-1} \mathcal{K}_{w}^{-1}\left(z^{*}, z\right) \partial / \partial z\left\{[\bar{\rho}(z) h(z)] /\left[N^{2}(z)\right]\right\}$, with $\bar{\rho}$ being the density and $\mathcal{K}_{w}$ the vertical velocity kernels introduced in appendix B.
} 
a) Eady model; large-scale rain, $r=\exp (-\mathrm{z} / 0.3)$

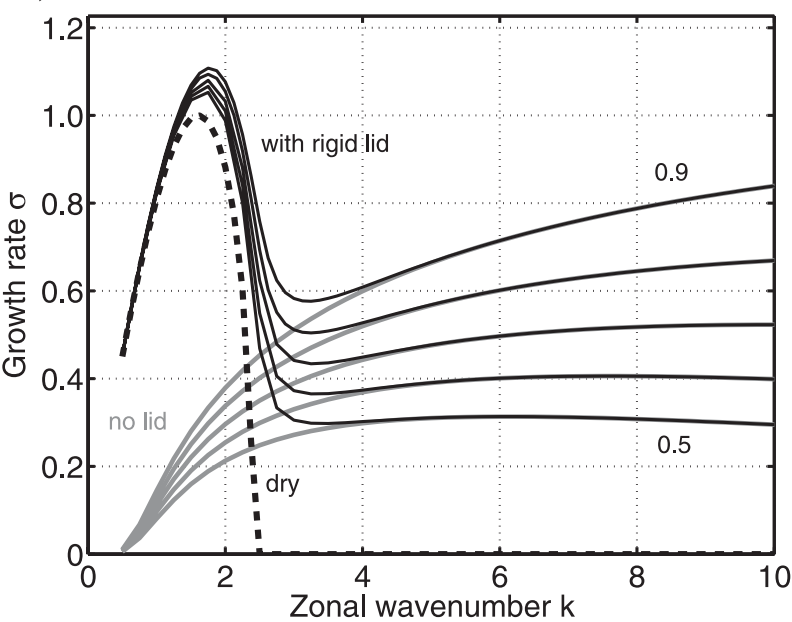

b) Eady model; large-scale rain, $r=\exp (-z / 0.3)$

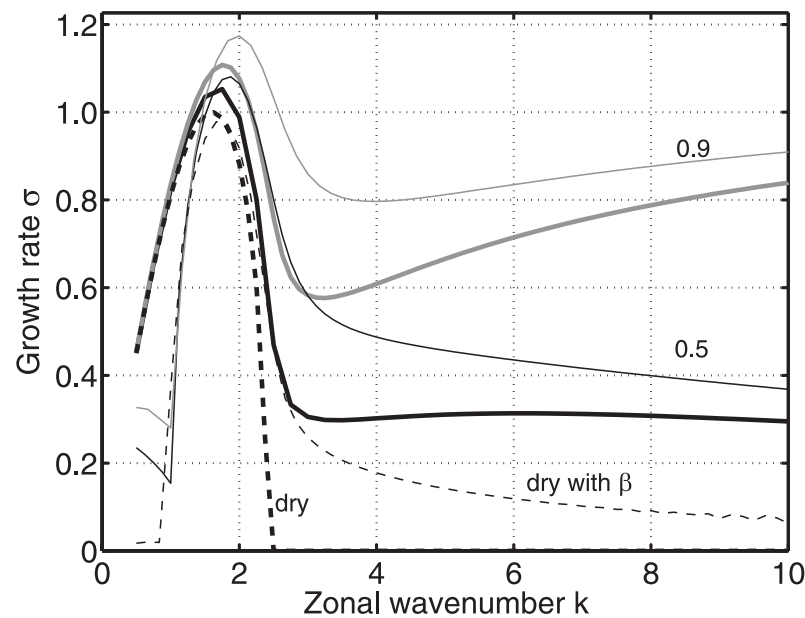

FIG. 7. (top) Dispersion relation for the Eady model $\left(z_{t}=10-\mathrm{km}\right.$ height) with large-scale rain parameterization and $\epsilon_{\mathrm{LSR}} \in(0.5,0.9)$. Gray lines show the results when no upper rigid boundary is present. (bottom) Dispersion relation for the Eady model with $\beta=0$ (heavy) and $\beta=1$ (thin) and large-scale rain for $\epsilon_{\mathrm{LSR}}=0$ (dashed), $\epsilon_{\mathrm{LSR}}=0.5$ (black), and $\epsilon_{\mathrm{LSR}}=0.9$ (gray).

\section{Large-scale rain examples}

Examples of baroclinic waves including the large-scale rain moisture parameterization are now explored. Attention is restricted to the semi-infinite and bounded Eady model, with or without $\beta$. The large-scale rain parameterization involves the specification of a moisture profile $r(z)$. Here we take the moisture profile to be exponentially decaying, $r(z)=\exp \left(-z / H_{m}\right)$, where $H_{m}$ is a moisture scale height (we take $H_{m}=0.3$ in units of $H$ ). Figure 7 shows the normal-mode dispersion relation for various values of $\epsilon_{\mathrm{LSR}}$ in the various domains. As has been noted in other studies (WD94; Moore and Montgomery 2004), the shortwave cutoff disappears, but even for
$\epsilon_{\mathrm{LSR}}=0.9$ the growth rate of the most unstable wave increases only by $10 \%-20 \%$. For a rapidly decreasing moisture profile one clearly needs to approach the limit of potential moist convective instability $\left(\epsilon_{\mathrm{LSR}} \rightarrow 1\right)$ to obtain growth rates that greatly exceed those of the dry Eady model. One implication for the atmosphere is that cases when baroclinic growth far outstrips that obtained from dry models require warm moist air through a greater depth and may need to be convectively unstable in the regions of saturated ascent such that deep convection can distribute latent heating through the troposphere. Figure 8 shows the classification diagrams for the Eady (1949) model, the Green (1960) model, and the Charney (1947) model with large-scale rain. Similar to the examples with cumulus heating (Fig. 4b), long waves with $k \leq 2$ (as well as shorter waves for small $\epsilon_{\mathrm{LSR}}$ ) mostly classify as DRY gNMs. For larger wavenumbers the gNMs all classify as diabatic Rossby waves, implying that there is a significant influence from the heating. Note that the other two classes Q2-Q1 (Snyder-Lindzen limit) and QT-Q1 (type-C cyclogenesis) do not occur with large-scale rain for any basic state. This is because the water vapor is concentrated in the lower troposphere so that heating layer is naturally near the ground where the boundary temperature wave of $q_{B}$ plays a major role. Also, in the Charney and Green models, which have an interior meridional PV gradient, the dry upper CRW can exist at any level since it is not confined to the tropopause as in the Eady model. As wavelength and the Rossby height scale are decreased, the upper CRWs (and therefore the dynamically induced vertical velocity) move closer to the ground, and consequently so does the heating maximum. The dry regime always exists at short wavelengths for sufficiently weak heating in models with $\beta$.

\section{General initial conditions}

The aim of this section is to show that when moisture is present, the general initial-value problem can be accurately predicted by considering just the four CRW components associated with the moist growing $\mathrm{NM}$ and a passively advected residual PV (PAR-PV). The starting point is Eqs. (19)-(21). V09 have shown that the dry adiabatic $(S=0)$ evolution from a broad range of initial conditions such as PV monopoles and dipoles is described accurately by attributing all the initial PV to the passive $q^{(p)}$ component and then integrating simple ODEs that describe the excitation of CRWs by the meridional velocity associated with $q^{(p)}$ and their subsequent propagation and interaction. The approach works because the displacement PV anomalies $q^{(d)}$ created through meridional advection project almost entirely onto a linear combination of the two CRW structures. The projection 

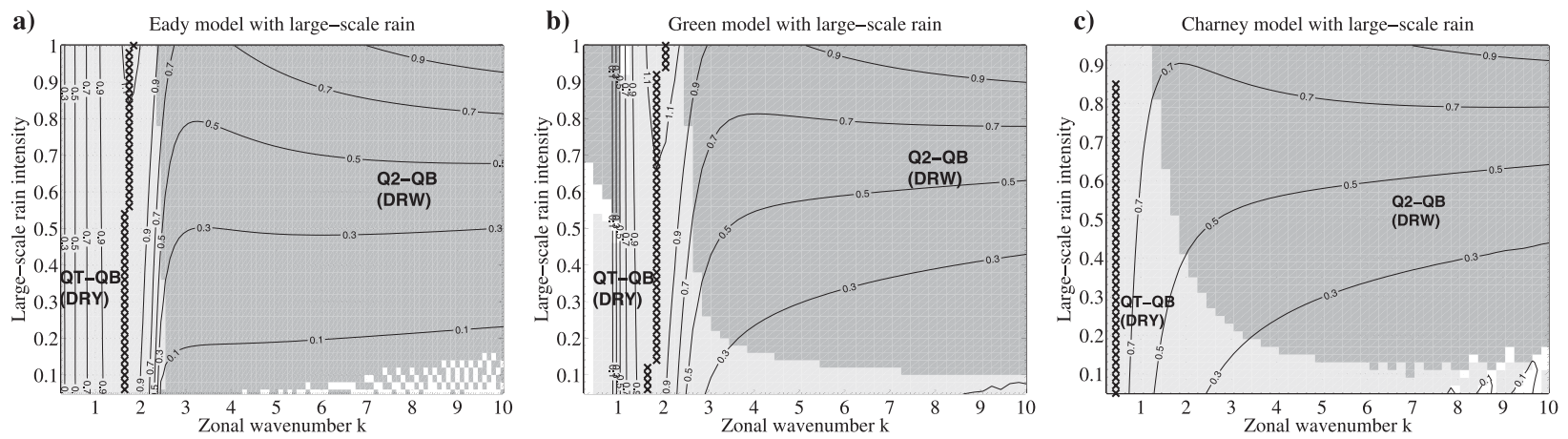

FIG. 8. Classification diagrams for baroclinic waves including the large-scale rain parameterization in the (left) Eady (middle) Green, and (right) Charney models. In case of the Eady and Green models, the rigid lid was located at $10 \mathrm{~km}$ height.

is not perfect and some additional displacement PV anomalies $q^{(n)}$ are generated. However, in dry models $q^{(n)}$ is orthogonal to both CRWs and therefore has no influence on their amplitude and phase evolution and also cannot influence $q^{(p)}$ (V09). V09 use the term "passively advected residual PV (PAR-PV) approximation" (p. 872) to describe the reduced model where $q^{(n)}$ is neglected, such that any PV not projected onto the CRWs is advected as if passive.

In problems with parameterized latent heating, the first step is to decompose the initial perturbation PV into three parts: $q(0)=q^{(d)}(0)+q^{(m)}(0)+q^{(p)}(0)$. Once $q^{(p)}(0)$ has been identified, its evolution is given by (21), simply "passive" advection with the basic-state wind $\bar{u}$. However, the meridional wind induced by $q^{(p)}$ is able to excite the two dry CRWs through meridional advection of basicstate PV. Its induced vertical motion also produces diabatic heating and the excitation of the two moist CRWs. The winds associated with $q^{(p)}$ may further project onto $\mathrm{PV}$, denoted as $q^{(n)}$, that cannot be attributed to the CRWs. Once $q^{(n)}$ is nonzero, it may further alter the dynamics of the four CRWs. The above sketched picture suggests that for general initial conditions the CRW evolution equations (24) contain two additional forcings:

$$
\dot{a}_{i}(t)=-i k\left[\sum_{j} c_{i j} a_{j}(t)+\mathcal{F}_{i}^{(p)}(t)+\mathcal{F}_{i}^{(n)}(t)\right],
$$

where $\mathcal{F}_{i}^{(p)}$ results from the passive RW component $q^{(p)}$ and is computed as

$$
\mathcal{F}_{i}^{(p)}(t)=\frac{\left\{q_{i}, \mathcal{N} \bar{\gamma} q^{(p)}\right\}}{\left\{q_{i}, \mathcal{N} q_{i}\right\}}
$$

and $\mathcal{F}_{i}^{(n)}(t)$ represents the forcing from $q^{(n)}$. However, unlike $\mathcal{F}_{i}^{(p)}$, the function $\mathcal{F}_{i}^{(n)}(t)$ is a priori unknown. For dry adiabatic evolution, it can be proved that $\mathcal{F}_{i}^{(n)}(t)=0$ at all times, even if $q^{(n)} \neq 0$ (V09). This arises because $q^{(n)}$ is orthogonal to the dry CRWs as mentioned above.
However, this is not true in general when including the heating parameterizations. Nevertheless, in systems with moisture the PAR-PV approximation is defined by neglecting $q^{(n)}$ at all times and equating the full evolution to the sum of the PAR-PV component $q^{(p)}$ and the four CRWs, as described by (26) with $\mathcal{F}_{i}^{(n)}(t)=0$.

\section{a. Evolution of a PV monopole in the presence of large-scale rain}

The previous discussion is illustrated with an example where the PAR-PV approximation reproduces the perturbation evolution quite accurately. A basic state is considered with smooth shear (positive in the troposphere, slightly negative in the stratosphere) and buoyancy frequency $(N=1$ in the troposphere, $N=2$ in the stratosphere), nonzero $\beta$, and with a density scale-height of $7.5 \mathrm{~km}$ (Fig. 9). Large-scale rain is included with a moisture profile that decays exponentially away from the surface with a height scale of $4 \mathrm{~km}$. We take $\epsilon_{\mathrm{LSR}}=1$, which is the largest value for which there is no potential moist convective instability in the lower troposphere (WD94). Because of the nontrivial basic state, the Green's functions are obtained numerically.

The initial condition takes the form of a midtropospheric PV monopole. The monopole is given a zonal wavelength of 0.8 of the wavelength of the most unstable dry Eady mode. The gNM growth rate at this wavelength is about $30 \%$ larger than that of the most unstable dry Eady mode and we expect the moist CRWs to contribute significantly to the evolution. Basic state and initial perturbation are shown in Fig. 9. Note how both the meridional wind and the vertical wind are in quadrature with the initial PV and how these fields quickly vanish in the stratosphere because of the higher stratification.

The KRW model (section 2e) was run forward to obtain the full PV, shown in the top row of Fig. 10. Already at $t=1$ the perturbation structure has changed significantly. A negative tropopause PV anomaly is generated at the location where the initial northward winds were maximal, 

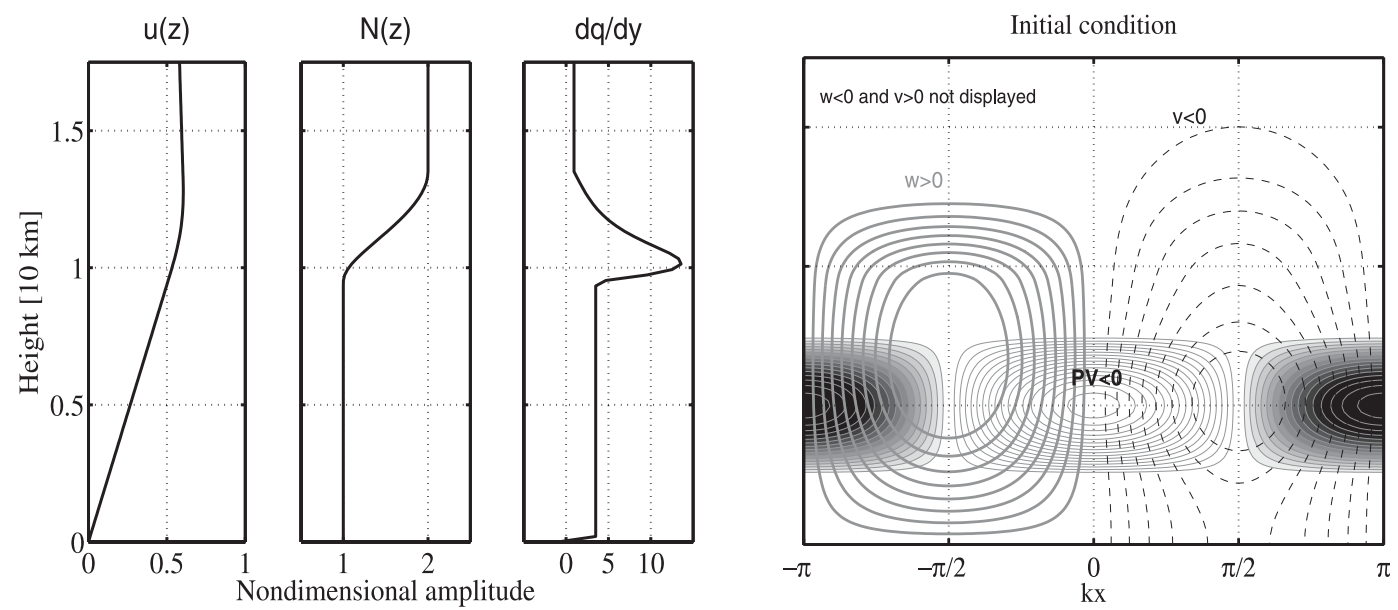

FIG. 9. Basic state and initial condition. Plotting conventions are as in Fig. 6. Note that $w<0$ and $v>0$ contours are not shown.

while below at the surface a warm potential temperature anomaly associated with positive boundary PV (black circles) appears. The tropopause-level negative PV induces an anticyclonic circulation, while the positive boundary PV induces a cyclonic circulation, such that the meridional wind (black contours) rapidly develops a westward tilt with height even though the initial PV tilts over eastward in the shear. Similarly, the vertical velocity (red contours) also develops a westward tilt. In time the perturbation gradually starts to resemble the gNM, which has a significant contribution from the low to midtropospheric PV.

The other rows in Fig. 10 show the partition of the full perturbation into RW components. The first row is the $q^{(p)}$ component, which equals the initial condition at $t=0$ and is simply sheared by the zonal flow. Note that its PV maximum is invariant, but the contour interval used in the plot increases exponentially at the gNM growth rate. Its meridional wind, and to a lesser extent vertical wind, tilt eastward with time reflecting the tilt in PV. The evolution is also shown in a frame moving with the gNM phase speed $\left(\sim 10 \mathrm{~m} \mathrm{~s}^{-1}\right)$, significantly slower than that of the midtropospheric basic state zonal wind, so the tilting PV pattern translates to the right.

The next two rows are the upper and lower dry CRWs, respectively. Both are untilted and amplify faster than the gNM growth rate, since their projection from the initial conditions is defined to be zero and they are excited through meridional advection associated with the PARPV component. A positive anomaly in $q^{(p)}$ induces cyclonic circulation, which advects basic-state positive PV from the north on its western side, especially at tropopause level. As a result the dry upper CRW is excited $\pi / 2$ upstream relative to the wave in $q^{(p)}$. The same positive $q^{(p)}$ anomaly advects warm air from the south on its eastern side, which at the ground is equivalent to a positive boundary PV anomaly (black dot in figure). Although the dry lower CRW contains a negative interior PV anomaly in the warm air, the positive boundary-PV anomaly immediately below is dominant in the inversion to obtain the winds such that the circulation is cyclonic around it. Consequently, the air to the east of the warm anomaly of the lower CRW moves poleward and ascends. The circulation associated with the upper and lower CRWs is therefore excited in antiphase, owing to the opposing PV gradients upon which they exist. In this phase the wind induced by one CRW at the level where the other is focused acts to hinder the propagation of the other CRW counter to the flow there. Consequently, the upper CRW is advected eastward relative to the lower CRW such that the westward tilt between them reduces with time.

In a similar way, the moist CRWs are excited by the vertical velocity associated with $q^{(p)}$. The moist upper CRW is excited in phase with the dry upper CRW. The moist lower CRW is in antiphase with it because the two moist CRWs exist in regions of opposing vertical gradient in heating (above and below the maximum). Although the structure of the two moist CRWs appears to have only a minor contribution to the full perturbation, it is found that their surface circulation has a major influence on the lower dry CRW and thus the whole evolution.

Note that the individual RW components were obtained by numerical integration of the reduced model (26) in time using the PAR-PV approximation $\left[\mathcal{F}_{i}^{(n)}(t) \rightarrow 0\right]$, where the initial condition was assigned entirely to the passive component $q^{(p)}$. The bottom row shows that part of the full model disturbance (top row) that is neglected under the PAR-PV approximation. Clearly it has its largest amplitude relative to the other components at intermediate times (it is zero initially and becomes small for $t>7$ ). The error made by making the PAR-PV approximation can be 
q

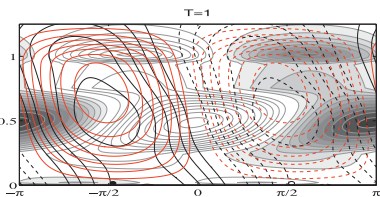

qp

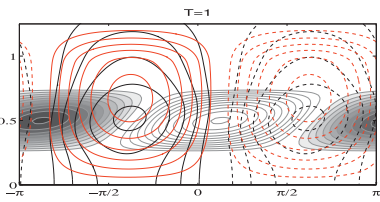

$T=1$

qT

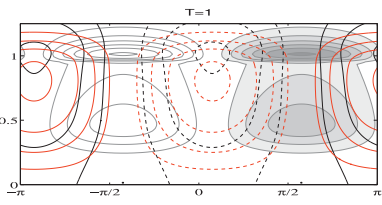

qB
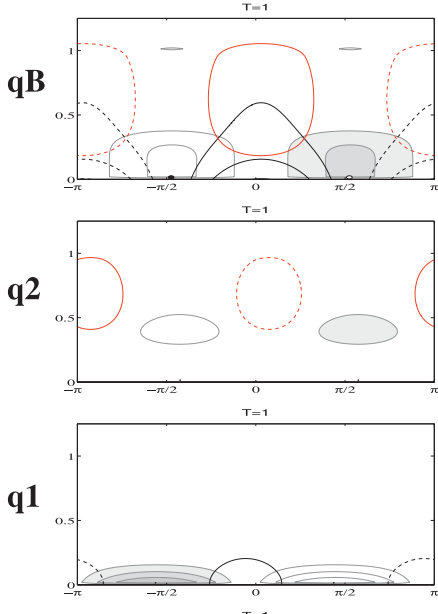

qn

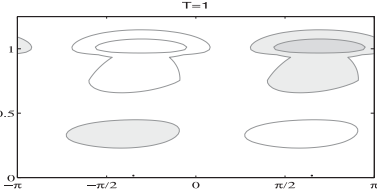

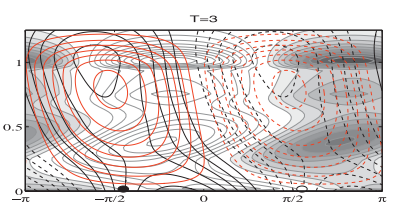

$\mathrm{T}=3$

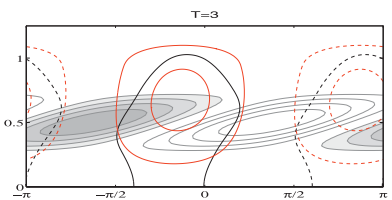

$\mathrm{T}=$
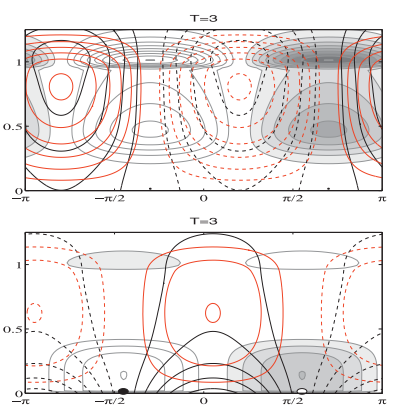

$\mathrm{T}=3$

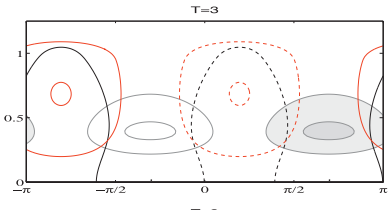

$\mathrm{T}=3$
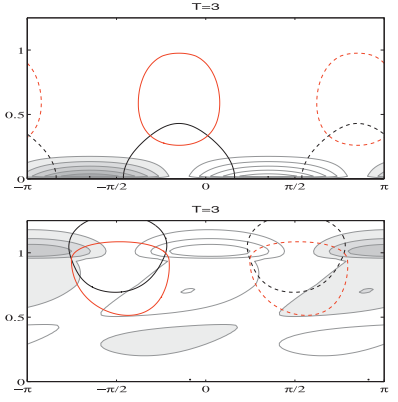

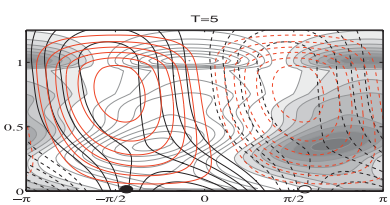

$T=5$
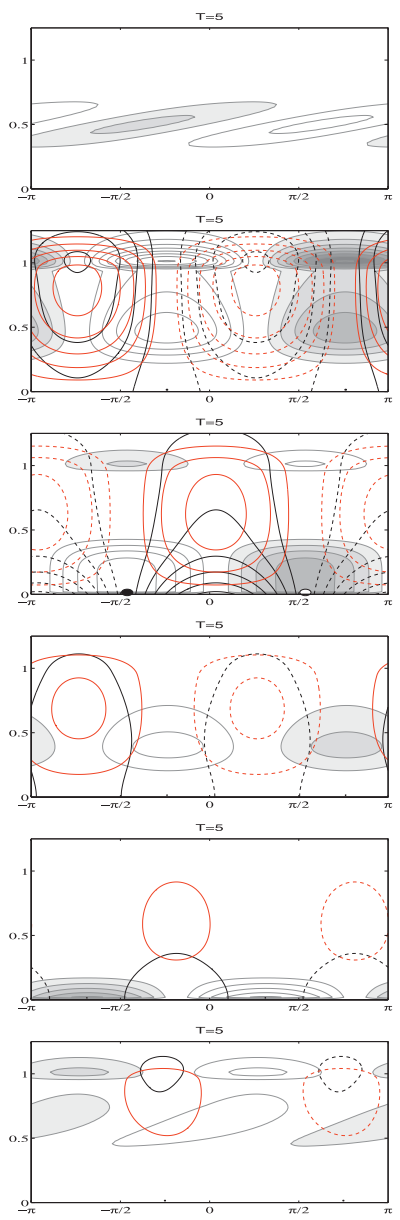
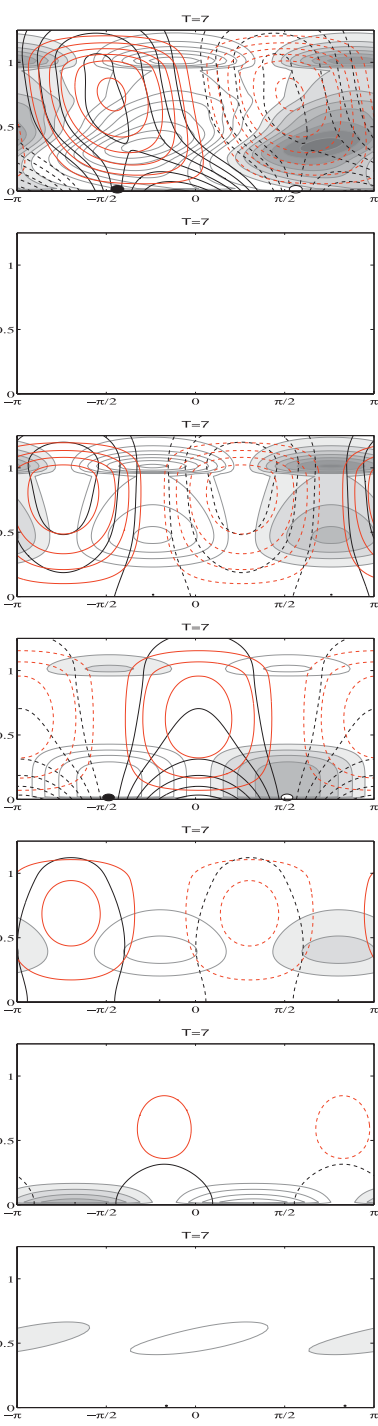

FIG. 10. Evolution of a PV monopole with large-scale rain parameterization on the basic state shown in Fig. 9. Viewed from frame of moving with the gNM phase speed. Plotting convention as in Fig. 6. The contour interval increases exponentially with $\sigma_{G}$. (top to bottom) Full PV $q, q^{(p)}, q_{T}, q_{B}, q_{2}, q_{1}$, and $q^{(n)}$ (the PV neglected by making the PAR-PV approximation). One time unit corresponds to approximately $10 \mathrm{~h}$ of physical time. The simulation starts at $T=0$ with all $\mathrm{PV}$ attributed to $q^{(p)}$.

measured as the maximal instantaneous total energy contained in the neglected perturbation $q^{(n)}$, which is about $3 \%$ of the full model total energy in this example.

\section{b. Phase space trajectories}

To examine the evolution of the RW components and in particular their propagation and interaction more quantitatively, we go back to the CRW evolution Eq. (26). By separating real and imaginary parts in (26), one obtains expressions for two key aspects of the CRWs: their instantaneous propagation rate $\dot{\epsilon}_{i}$ and growth rate $\dot{A}_{i} / A_{i}$. Assuming the PAR-PV approximation (setting $\mathcal{F}_{i}^{(n)}=0$ ), one obtains $\dot{\epsilon}_{i}=-k\left[\sum_{j} c_{i j} \frac{A_{j}}{A_{i}} \cos \left(\epsilon_{i}-\epsilon_{j}\right)+\frac{F_{i}^{(p)}}{A_{i}} \cos \left(\epsilon_{i}-\eta_{i}^{p}\right)\right]$

and

$$
\frac{\dot{A}_{i}}{A_{i}}=-k\left[\sum_{j} c_{i j} \frac{A_{j}}{A_{i}} \sin \left(\epsilon_{i}-\epsilon_{j}\right)+\frac{F_{i}^{(p)}}{A_{i}} \sin \left(\epsilon_{i}-\eta_{i}^{p}\right)\right],
$$

where $F_{i}^{(p)}=\left|\mathcal{F}_{i}^{(p)}\right|$ and $\eta_{i}^{p}=\arg \left[\mathcal{F}_{i}^{(p)}\right]$. The evolution of CRW- $i$ can be represented as a trajectory $Z_{i}(t)=$ $\left[X_{i}(t), Y_{i}(t)\right]=\left[\dot{\epsilon}_{i}, \dot{A}_{i} / A_{i}\right]$ in the two-dimensional "propagation rate $X$ growth rate" plane. Since both growth and 


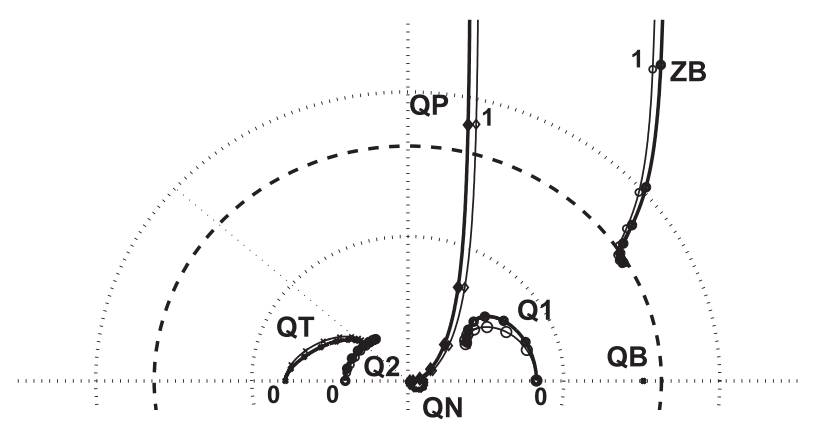

FIG. 11. Phase-space trajectory (ZB) of the lower dry CRW and the contributions to its zonal propagation ( $x$ axis is $\dot{\epsilon})$ and growth rate ( $y$ axis is $\dot{\mathrm{A}} / \mathrm{A}$ ) from itself $(\mathrm{QB})$ and the other $\mathrm{RW}$ components (QP, QT, Q1, Q2, and QN, which is neglected in the PAR-PV approximation). Thick lines indicate the trajectories of the full model, thin lines those of the reduced (PAR-PV) model. Numbers indicate nondimensional times. Dots are separated by one nondimensional time unit. The inner and outer dotted circles have radii of 0.5 and 1.0 , respectively; the dashed circle has a radius $|k c|=\left|k c_{r}+i \sigma\right|($ in units of $f \Lambda / N)$.

propagation rate asymptote to constant values, the CRW trajectories will "end" at a fixed point. The evolution of each CRW (i.e., the trajectory in propagation rate-growth rate space) results from CRW self-propagation and from the interactions with the other RW components [all terms on the rhs of (27) and (28)]. The contributions of all these interactions can be compared quantitatively by displaying them as separate trajectories in the same figure. By zooming in on the evolution of the individual CRWs, the trajectory analysis further deepens the understanding of the perturbation evolution.

For the lower dry CRW, the trajectory is labeled ZB in Fig. 11. Also shown is the trajectory of the lower dry CRW, as obtained with the full model [i.e., by projecting the full $q^{(d)}$ obtained from (19) onto the lower CRW]. In this example the trajectories are very similar but not identical. The CRW evolution is most easily understood by analyzing the separate contributions. First of all, QB denotes the self-contribution of the CRW. As for all vertically untilted PV waves, it does not contribute to its own growth but it has an important contribution to its phase propagation (eastward propagation), which is smaller than $f \Lambda / N$ of a pure boundary edge wave, due to the presence of interior $\mathrm{PV}$ of opposing sign in the lower CRW's PV structure. The contribution from the passive component $(\mathrm{QP})$ to the CRW's growth rate dominates the growth rate for times $t \leq 2.5$ (it excites the lower CRW $\pi / 2$ downstream at $t=0$ ) while also adding to the eastward propagation rate of the CRW. Its influence, however, rapidly decreases, both because the $q_{p}$ structure itself is in the decaying phase of the Orr mechanism and because the other components amplify rapidly. Interestingly, Q1, the lower moist CRW, always helps the propagation of $\mathrm{QB}$ and promotes its growth. (Since both dry and moist lower CRWs propagate as on a negative PV gradient, the former statement implies that the lower dry CRW acts to reduce the growth of Q1 at all times). Q2 and QT act in concert to hinder the propagation rate of $\mathrm{QB}$ and promote its growth by the same amount in the gNM configuration. Also, their combined hindering behavior is almost cancelled out by the helping effect of Q1 such that the net speed of the lower dry CRW is not much smaller than its self-propagation rate (QB).

The trajectories obtained by making the PAR-PV approximation are almost identical to those of the full model, implying that neglecting the PV not involved with the components is justified.

\section{Conclusions and remarks}

This paper describes how the Rossby wave (RW) perspective of baroclinic instability (e.g., Hoskins et al. 1985; H04; Methven and De Vries 2008; V09) can be extended to include diabatic generation of $\mathrm{PV}$ resulting from simple moisture parameterizations. Two different types of moisture parameterizations (wave-CISK and large-scale rain) have been included in the QG system for basic states of varying complexity. Although realistically the parameterizations should be conditional on ascent, in this paper the parameterizations are taken in unconditional (linearized) form. The nonlinear problem will be addressed in future work (Frame et al. 2010, unpublished manuscript).

To understand baroclinic instability with diabatic heating from moisture parameterizations, it is important to realize that $\mathrm{PV}$ anomalies can have three different origins. PV anomalies can be created through advection of the basic-state PV contours in regions where the mean meridional potential vorticity gradient $\bar{q}_{y} \neq 0$. This is called "displacement PV," $q^{(d)}$. Unstable normal modes in the absence of moisture are fully described by such displacement PV. PV anomalies can also be generated diabatically through active moist processes, in which case it is referred to as "moist PV," $q^{(m)}$. Finally, there can be PV anomalies that are remnants of diabatic processes, which are no longer active. This PV is called "passive PV," $q^{(p)}$, because it is simply advected by the basic state wind $\bar{u}$. V09 have presented an RW framework for interpreting baroclinic development where the PV distribution contains both displacement PV $q^{(d)}$ and passive $\mathrm{PV} q^{(p)}$, but where the diabatic PV generating processes were absent.

This work explicitly includes moist PV $q^{(m)}$ and details a RW framework where all PV components are present. Four RW components are used to interpret baroclinic instability of moist normal modes. Two of these are similar to the counterpropagating Rossby waves (CRWs) appearing in the dry theory (H04). The other two components 
are defined from the moist PV that can be attributed exclusively to the moist processes. The main advantage of using RW components to describe the flow is that it gives a robust framework for analysis. The dynamics is first reduced to one PDE by relating vertical velocity to the PV distribution and then reduced to four coupled ODEs describing the amplitude and phase evolution of the RW components. Based on the occurrence of mutually amplifying pairs of RW components, four growth regimes have been defined, ranging from essentially dry baroclinic instability (two dry CRWs dominate the interaction) to fully moist (two moist CRWs dominate; SL91), with a diabatic Rossby wave and type-C cyclone growth being intermediate types. The RW framework makes transparent that instability and sustained mutual amplification can only occur if the RW components (whether dry or moist) propagate in opposite directions to one another and counter to the shear in the zonal flow (cf. Hayashi and Young 1987).

The RW components have further been used in the interpretation of the baroclinic initial value problems with moisture. General initial conditions involve PV of all three origins: displacement, diabatic, and passive. A reduced model can be constructed based on the four RW components and a passive component in an approach which has previously been termed the passively advected residual PV approximation (PAR-PV approximation; V09). The reduced model captures the main propagation and interaction mechanisms available to the system and accurately describes the evolution for many initial conditions. The evolution can be succinctly summarized in terms of the trajectories of the components in phase space. The trajectories obtained from the reduced model are almost identical to those from the full system projected onto the RW components. The reduced model approach, however, has caveats similar to those described in V09 but can be expected to work well in cases in which the underlying normal modes have reasonably large growth rates.

The present paper discusses a partitioning into four CRW components. This number is motivated physically by the partitioning, first by process into the displacement and diabatic PV anomalies and second by the requirement for two orthogonal structures in each (necessary for modal growth).

Finally, by defining the classification, we are making explicit the relative importance of the different interactions and processes within the flow, and determining their distributions in parameter space. Despite the fact that the present study is inherently oversimplifying many aspects of moist baroclinic instability occurring in the real atmosphere, it is hoped that in this way the diabatic Rossby wave moves from being a concept used in case studies (e.g., Wernli et al. 2002) to being something that might be expected to be observed in a certain region of parameter space.

Acknowledgments. All authors acknowledge the National Environmental Research Council (NERC, Grant $\mathrm{NE} / \mathrm{D} 011507 / 1$ ) for making the research possible. HdV acknowledges the Netherlands Organisation for Scientific Research (NWO) for a Rubicon Grant. JM is grateful for a Research Council UK Academic Fellowship. The paper has benefited from comments by Mankin Mak and another reviewer.

\section{APPENDIX A}

\section{Inverting the Omega Equation with Large-Scale Rain}

This appendix extends section $2 \mathrm{~d}$ by including largescale rain. As in the main text a Green function method is used. First, in (6) the term proportional to $\epsilon_{\mathrm{LSR}}$ is moved from the rhs to the lhs. A (new) Green function $\tilde{\mathcal{G}}_{w}$ is constructed for the (new) differential operator $\tilde{\mathcal{L}_{w}} \equiv \mathcal{L}_{w}-\epsilon_{\mathrm{LSR}} r(z) \nabla_{H}^{2}$. Using $\tilde{\mathcal{G}_{w}}$ instead of $\mathcal{G}_{w}$, one can repeat the steps as in section $2 \mathrm{~d}$. The result is [cf. (8)]

$$
w(z, t)=\tilde{w}^{(d)}(z, t)+\tilde{w}^{(h)}(z, t),
$$

where the two contributions are now given by

$$
\begin{aligned}
& \tilde{w}^{(d)}(z, t)=\int \tilde{\mathcal{G}}_{w}\left(z, z^{\prime}, \epsilon_{\mathrm{LSR}}\right) F^{(d)}\left(z^{\prime}, t\right) d z^{\prime} \text { and } \\
& \tilde{w}^{(h)}(z, t)=\frac{\epsilon_{\mathrm{CCV}} \tilde{w}^{(d)}(z *, t)}{1-\epsilon_{\mathrm{CCV}} \tilde{\alpha}\left(z *, \epsilon_{\mathrm{LSR}}\right)} \tilde{\alpha}\left(z, \epsilon_{\mathrm{LSR}}\right)
\end{aligned}
$$

with $\tilde{\alpha}\left(z, \epsilon_{\mathrm{LSR}}\right)=-\left(k^{2}+l^{2}\right) \int \tilde{\mathcal{G}}_{w}\left(z, z^{\prime}, \epsilon_{\mathrm{LSR}}\right) h\left(z^{\prime}\right) d z^{\prime}$. Obviously, $\tilde{\mathcal{G}}_{w}$ depends explicitly on the LSR moisture intensity parameter $\epsilon_{\mathrm{LSR}}$ and the moisture profile $r(z)$. Therefore, although $\tilde{w}^{(d)}$ has been given the superscript $d$ [because of its similarity to (9)], it is influenced by the LSR heating parameterization. In some cases, $\tilde{\mathcal{G}}_{w}$ can be obtained analytically. For a uniform moisture profile, $\tilde{\mathcal{G}_{w}}$ is described by hyperbolic functions. If the moisture profile decays exponentially with height, $\tilde{\mathcal{G}}_{w}$ is expressible in terms of Bessel functions. For more general moisture profiles, one will have to use numerical methods. Note that $\tilde{w}^{(h)}=0$ if $\epsilon_{\mathrm{CCV}}=0$, and that $\epsilon_{\mathrm{CCV}} \tilde{\alpha}\left(z_{*}, \epsilon_{\mathrm{LSR}}\right)=1$ again defines a CISK threshold [which now depends on $k, h(z), r(z), z_{*}$, and $\epsilon_{\mathrm{CCV}}$ and $\left.\epsilon_{\mathrm{LSR}}\right]$. 


\section{APPENDIX B}

\section{Derivation of (13) Using Kernel Rossby Wave Theory}

Following Heifetz and Methven (2005), the starting point is to write the PV as a continuous integral over Rossby wave kernels, called kernel Rossby waves (KRWs). KRWs can simply be thought of as being unique objects, which have associated with them various "attributable fields," such as PV, streamfunction, etc. Central to the KRW definition is its $\mathrm{PV}$ distribution, denoted as $\mathcal{K}_{q}\left(z, z^{\prime}\right)$, which takes the form of a $\delta$ function:

$$
\mathcal{K}_{q}\left(z, z^{\prime}\right)=\delta\left(z-z^{\prime}\right)
$$

All KRWs are labeled uniquely by the vertical position of their PV. KRWs are orthogonal in PV such that they form a proper basis. Associated with the KRW, or "induced" by its PV, are a streamfunction and a meridional and vertical velocity distribution, denoted as $\mathcal{K}_{\psi}\left(z, z^{\prime}\right)$, $\mathcal{K}_{v}\left(z, z^{\prime}\right)$, and $\mathcal{K}_{w}\left(z, z^{\prime}\right)$ respectively.

The KRW PV induces a streamfunction $\mathcal{K}_{\psi}\left(z, z^{\prime}\right)$ that satisfies $\mathcal{L}_{\psi}\left[\mathcal{K}_{\psi}\left(z, z^{\prime}\right)\right]=\mathcal{K}_{q}\left(z, z^{\prime}\right)$. Therefore $\mathcal{K}_{\psi}\left(z, z^{\prime}\right)$ is simply given by the Green function $\mathcal{G}_{\psi}\left(z, z^{\prime}\right)$. By integrating over all kernels with the appropriate amplitudes the streamfunction is recovered; that is, $\psi(z, t)=\int \mathcal{K}_{\psi}\left(z, z^{\prime}\right) q\left(z^{\prime}, t\right) d z^{\prime}$. In the same spirit each $\mathrm{KRW}$ is assigned a meridional velocity density $\mathcal{K}_{v}\left(z, z^{\prime}\right)$, and $v$ is written as

$$
v(z, t)=\int \mathcal{K}_{v}\left(z, z^{\prime}\right) q\left(z^{\prime}, t\right) e^{-i \pi / 2} d z^{\prime},
$$

where $\mathcal{K}_{v}\left(z, z^{\prime}\right)=k \mathcal{G}_{\psi}\left(z, z^{\prime}\right)$. The factor $\exp (-i \pi / 2)$ is merely convention: it guarantees that the meridional velocity kernel $\mathcal{K}_{v}\left(z, z^{\prime}\right)$ is real and positive definite.
As shown in section $2 \mathrm{~d}$, the linearized omega equation (6) can be inverted to obtain the vertical velocity. The dynamical "forcing" appearing in $(6), F^{(d)}$, was shown in (7) to be expressible entirely in terms of $v, F^{(d)}=\mathcal{L}_{\text {lin }}[v]$. By substituting for $v$ the KRW velocity distribution $\mathcal{K}_{v}\left(z, z^{\prime}\right)$ and defining $\hat{F}^{(d)}\left(z, z^{\prime}\right) \equiv \mathcal{L}_{\operatorname{lin}}\left[\mathcal{K}_{v}\left(z, z^{\prime}\right)\right]$, the "dynamic" part of the vertical velocity, $w^{(d)}(z)$, can be written as

$$
w^{(d)}(z, t)=\int \mathcal{K}_{w}^{(d)}\left(z, z^{\prime}\right) q\left(z^{\prime}, t\right) e^{-i \pi / 2} d z^{\prime},
$$

where $\mathcal{K}_{w}^{(d)}\left(z, z^{\prime}\right)=\int \mathcal{G}_{w}\left(z, z^{\prime \prime}\right) \hat{F}^{(d)}\left(z^{\prime \prime}, z^{\prime}\right) d z^{\prime \prime} ; \mathcal{G}_{w}$ is the Green function corresponding to the operator $\mathcal{L}_{w}$ appearing in (6). [If LSR also occurs, one needs a decomposition of $\tilde{w}^{(d)}(\mathrm{A} 2)$, which follows by replacing $\mathcal{G}_{w}$ by $\tilde{\mathcal{G}}_{w}$ in (B3) above]. It was further shown that selfconsistency leads to a relation between $w^{(h)}$ and $w^{(d)}$, suggesting that the total vertical velocity $w$ can also be written as

$$
w(z, t)=\int \mathcal{K}_{w}\left(z, z^{\prime}\right) q\left(z^{\prime}, t\right) e^{-i \pi / 2} d z^{\prime}
$$

for some kernel $\mathcal{K}_{w}\left(z, z^{\prime}\right)$ (not shown). Using $\mathcal{K}_{v}$ and $\mathcal{K}_{w}$, the PV equation reads

$$
\frac{\partial q}{\partial t}=-i k \tilde{\mathcal{A}}[q], \tilde{\mathcal{A}}[q]=\bar{u}(z) q(z)-\frac{1}{k} \int \gamma\left(z, z^{\prime}\right) q\left(z^{\prime}\right) d z^{\prime},
$$

where the interaction coefficients $\gamma\left(z, z^{\prime}\right)=\gamma^{(d)}\left(z, z^{\prime}\right)+$ $\gamma^{(m)}\left(z, z^{\prime}\right)$ are defined as

$$
\gamma^{(d)}\left(z, z^{\prime}\right)=\frac{\partial \bar{q}(z)}{\partial y} \mathcal{K}_{v}\left(z, z^{\prime}\right) \text { and }
$$

$$
\gamma^{(m)}\left(z, z^{\prime}\right)=\frac{\epsilon_{\mathrm{CCV}}}{\bar{\rho}(z)} \frac{\partial}{\partial z}\left[\frac{-\bar{\rho}(z) h(z) \mathcal{K}_{w}\left(z *, z^{\prime}\right)}{N^{2}(z)}\right]+\frac{\epsilon_{\mathrm{LSR}}}{\bar{\rho}(z)} \frac{\partial}{\partial z}\left[\frac{-\bar{\rho}(z) r(z) \mathcal{K}_{w}\left(z, z^{\prime}\right)}{N^{2}(z)}\right]
$$

Equation (B5) is equal to (13). Note that because $\mathcal{K}_{w}$ contains both $\epsilon_{\mathrm{CCV}}$ and $\epsilon_{\mathrm{LSR}}$ (if both parameterizations act simultaneously), it is not possible to separate contributions from large-scale rain and wave-CISK.

\section{REFERENCES}

Bretherton, F. P., 1966: Baroclinic instability and the short wavelength cut-off in terms of potential vorticity. Quart. J. Roy. Meteor. Soc., 92, 335-345.
Charney, J. G., 1947: The dynamics of long waves in a baroclinic westerly current. J. Atmos. Sci., 4, 136-162.

— jets in a rotating atmosphere. J. Atmos. Sci., 19, 159-172.

Craig, G., and H.-R. Cho, 1988: Cumulus heating and CISK in the extratropical atmosphere. Part I: Polar lows and comma clouds. J. Atmos. Sci., 45, 2622-2640.

Davies, H. C., and C. H. Bishop, 1994: Eady edge waves and rapid development. J. Atmos. Sci., 51, 1930-1946.

De Vries, H., and J. D. Opsteegh, 2007: Resonance in optimal perturbation evolution. Part I: Two-layer Eady model. J. Atmos. Sci., 64, 673-694. 
—, J. Methven, T. H. A. Frame, and B. J. Hoskins, 2009: An interpretation of baroclinic initial value problems: Results for simple basic states with non-zero interior PV gradients. J. Atmos. Sci., 66, 864-882.

Eady, E. T., 1949: Long waves and cyclone waves. Tellus, 1, 33-52.

Emanuel, K. A., M. Fantini, and A. J. Thorpe, 1987: Baroclinic instability in an environment of small stability to slantwise moist convection. Part I: Two-dimensional models. J. Atmos. Sci., 44, 1559-1573.

Fjørtoft, R., 1950: Application of integral theorems in deriving criteria of stability for laminar flows and for the baroclinic circular vortex. Geofys. Publ., 17, 1-52.

Gray, S. L., 2006: Mechanisms of midlatitude cross-tropopause transport using a potential vorticity budget approach. J. Geophys. Res., 111, D17113, doi:10.1029/2005JD006259.

Green, J. S. A., 1960: A problem in baroclinic stability. Quart. J. Roy. Meteor. Soc., 86, 237-251.

Gyakum, J. R., 1983: On the evolution of the $Q E$ II storm. II: Dynamic and thermodynamic structure. Mon. Wea. Rev., 111, 1156-1173.

Hayashi, Y. Y., and W. R. Young, 1987: Stable and unstable shear modes of rotating parallel flows in shallow water. J. Fluid Mech., 184, 477-504.

Heifetz, E., and J. Methven, 2005: Relating optimal growth to counterpropagating Rossby waves in shear instability. Phys. Fluids, 17, 064107, doi:10.1063/1.1937064.

, C. H. Bishop, B. J. Hoskins, and J. Methven, 2004a: The counter-propagating Rossby-wave perspective on baroclinic instability. I: Mathematical basis. Quart. J. Roy. Meteor. Soc., 130, 211-231.

— counter-propagating Rossby-wave perspective on baroclinic instability. II: Application to the Charney model. Quart. J. Roy. Meteor. Soc., 130, 233-258.

Held, I. M., 1985: Pseudomomentum and the orthogonality of modes in shear flows. J. Atmos. Sci., 42, 2280-2288.

Hoskins, B. J., M. E. McIntyre, and A. W. Robertson, 1985: On the use and significance of isentropic potential vorticity maps. Quart. J. Roy. Meteor. Soc., 111, 877-946.

- M. Pedder, and D. W. Jones, 2003: The omega equation and potential vorticity. Quart. J. Roy. Meteor. Soc., 129, 3277-3303.
Kuo, Y.-H., M. A. Shapiro, and E. G. Donall, 1991: The interaction between baroclinic and diabatic processes in a numerical simulation of a rapidly intensifying extratropical marine cyclone. Mon. Wea. Rev., 119, 368-384.

Lindzen, R. S., 1974: Wave-CISK in the tropics. J. Atmos. Sci., 31, $156-179$.

Mak, M., 1982: On moist quasi-geostrophic baroclinic instability. J. Atmos. Sci., 39, 2028-2037.

_ 1994: Cyclogenesis in a conditionally unstable moist baroclinic atmosphere. Tellus, 46A, 14-33.

_ , and P. R. Bannon, 1984: Frontogenesis in a moist semigeostrophic model. J. Atmos. Sci., 41, 3485-3500.

Methven, J., and H. De Vries, 2008: Comments on "Piecewise potential vorticity inversion: Elementary tests." J. Atmos. Sci., 65, 3003-3008.

Montgomery, M. T., and B. F. Farrell, 1991: Moist surface frontogenesis associated with interior potential vorticity anomalies in a semigeostrophic model. J. Atmos. Sci., 48, 343-367.

Moore, R. W., and M. T. Montgomery, 2004: Reexamining the dynamics of short-scale, diabatic Rossby waves and their role in midlatitude moist cyclogenesis. J. Atmos. Sci., 61, 754-768.

Orr, W., 1907: Stability or instability of the steady motions of a perfect liquid. Proc. Roy. Irish Acad., 27, 9-138.

Parker, D. J., and A. J. Thorpe, 1995: Conditional convective heating in a baroclinic atmosphere: A model of convective frontogenesis. J. Atmos. Sci., 52, 1699-1711.

Plant, R. S., G. C. C. Craig, and S. L. Gray, 2003: On a three-fold theory of extratropical cyclogenesis. Quart. J. Roy. Meteor. Soc., 129, 2989-3012.

Raymond, D. J., and H. Jiang, 1990: A theory for long-lived mesoscale convective systems. J. Atmos. Sci., 47, 3067-3077.

Sen, S. K., and V. C. Venkaiah, 1988: On symmetrizing a matrix. Indian J. Pure Appl. Math., 19, 554-561.

Snyder, C., and R. S. Lindzen, 1991: Quasi-geostrophic wave-CISK in an unbounded baroclinic shear. J. Atmos. Sci., 48, 76-86.

Wernli, H., S. Dirren, M. A. Liniger, and M. Zillig, 2002: Dynamical aspects of the life cycle of the winter storm 'Lothar' (24-26 December 1999). Quart. J. Roy. Meteor. Soc., 128, 405-429.

Whitaker, J. S., and C. A. Davis, 1994: Cyclogenesis in a saturated environment. J. Atmos. Sci., 51, 889-907. 